\title{
Spontaneous Generation of Inertia-Gravity Waves during the Merging of Two Baroclinic Anticyclones
}

\author{
EnRic Pallàs-Sanz and Álvaro Viúdez \\ Institut de Ciències del Mar, CSIC, Barcelona, Spain
}

(Manuscript received 19 October 2006, in final form 6 March 2007)

\begin{abstract}
The spontaneous generation and propagation of short-scale inertia-gravity waves (IGWs) during the merging of two initially balanced (void of IGWs) baroclinic anticyclones is numerically investigated. The IGW generation is analyzed in flows with different potential vorticity (PV) anomaly, numerical diffusion, numerical resolution, vortex aspect ratio, and background rotation. The vertical velocity and its vertical derivative are used to identify the IGWs in the total flow, while the unbalanced flow (the waves) is diagnosed using the optimal PV balance approach. Spontaneous generation of IGWs occurs in all the cases, primarily as emissions of discrete wave packets. The increase of both the vortex strength and vortex extent isotropy enhances the IGW emission. Three possible indicators, or theories, of spontaneous IGW generation are considered, namely, the advection of PV, the material rate of change of the horizontal divergence, and the three-dimensional baroclinic IGW generation analogy of Lighthill sound radiation theory. It is suggested that different mechanisms for spontaneous IGW generation may be at work. One mechanism is related to the advection of PV, with the IGWs in this case having wave fronts similar to the PV isosurfaces in the upper layers, and helical patterns in the deep layers. Trapped IGWs are ubiquitous in the vortex interior and have annular wave front patterns. Another mechanism is related to the spatially coherent motion of preexisting IGWs, which eventually cooperate to produce mean flow, in particular larger-scale horizontal divergence, and therefore larger-scale vertical motion, which in turns triggers the emission of new IGWs.
\end{abstract}

\section{Introduction}

In stratified rotating flows, such as those in the atmosphere and ocean, inertia-gravity waves (IGWs; short scale and large phase speed) coexist simultaneously with the balanced flow (large scale and short phase speed). Generation of IGWs may be primarily caused by initial disturbances on the otherwise balanced (void of IGWs) flow, as for example those due to wind stress or bottom topography. As these waves, produced at the ocean boundaries, propagate into the ocean interior, some energy is transferred to higher vertical wavenumbers, causing a greater vertical shear that favors wave breaking and hence generating small patches of turbulence (Garrett 1979; Thorpe 1999; Alford and Pinkel 2000). The eventual breaking of IGWs

Corresponding author address: Enric Pallàs-Sanz, Institut de Ciències del Mar, CSIC, P. Marítim de la Barceloneta, 37-49 08003-Barcelona, Spain.

E-mail: epallas@cmima.csic.es dissipates the gradients produced by larger-scale motions, partially driving the thermohaline circulation (Garrett 2003). IGWs easily develop also when unbalanced initial conditions are used in numerical simulations (e.g., Daley 1991, chapter 6); meanwhile the unbalanced mass and momentum fields slowly adjust through geostrophic adjustment (Rossby 1938) or, more generally, any balance adjustment (Zhang 2004). Recently, several oceanographic and atmospheric studies (Ford 1994; Ford et al. 2000; Afanasyev 2003; Lovegrove et al. 2000; Plougonven and Zeitlin 2002; Williams et al. 2003, 2005; Lane et al. 2004; Viúdez and Dritschel 2006; Viúdez 2006) have shown that weak (in the horizontal velocity field) IGWs can be spontaneously emitted (i.e., in the absence of any external forcing like wind stress or bottom topography) from balanced or near-balanced, nonstationary flows.

This particular case of spontaneous emission of IGWs is referred to as Lighthill-Ford radiation because Ford extends the Lighthill theory of aerodynamic sound generation (Lighthill 1952) to the shallow water

DOI: 10.1175/2007JPO3716.1 
dynamics (Ford 1994), or as spontaneous-adjustment emission (SAE), which seems more appropriate since the mechanism of the spontaneous generation of IGWs is not yet fully understood. The SAE thus questions the existence of the strict slow manifold (Leith 1980; Lorenz 1986; Jacobs 1991) in favor of the slow quasi manifold (Ford et al. 2000). The spontaneous IGWs can be energetic enough to contribute as an interior source (far from the ocean's boundaries) to the energy budget of the gravity wave spectra in the ocean. Afanasyev (2003) found that approximately $4 \%$ of the total flow energy is radiated during the adjustment after a secondary vortex dipole collision in a rotating stratified tank. SAE represents a new source of IGWs that could explain the gap of the total amount of energy going into the IGWs' pool, usually computed only considering the wind stress and internal tides' inputs. Here, we investigate numerically the spontaneous generation of IGWs during the merging of two corotating mesoscale baroclinic vortices on the $f$ plane.

The initial generation of IGWs in numerical simulations is a physical process caused by special (unbalanced) initial conditions which, though considered unrealistic, are used because the initial balanced fields are often unknown. Several methods can be applied to filter out these initial IGWs and to obtain a balanced or near-balanced description of the flow. The quasigeostrophic (e.g., Daley 1991, chapter 7) and semigeostrophic (Hoskins 1975) dynamics prohibit this generation but they may filter an important part of the balanced three-dimensional flow as well (Viúdez and Dritschel 2004a). The nonlinear normal-mode initialization (Machenhauer 1977; Baer and Tribbia 1977) effectively defines a slow manifold without permitting spontaneous IGW generation by setting to zero gravity wave modes. Filtering techniques, such as the digital filter initialization (Lynch and Huang 1992; Huang and Lynch 1993), have been also used to avoid initial IGWs. In this work we used the potential vorticity (PV) initialization procedure (Viúdez and Dritschel 2003, hereafter VD03) based on the slow, progressive growth of the PV field, in a Lagrangian way, during a given initialization time $\Delta t_{I}$.

The shallow water model, the simplest system permitting both gravity and Rossby waves, has been often used to investigate spontaneous IGWs. Ford (1994) investigates the relation of the gravity waves, radiated from a shallow water vortex train, with the Froude $(\mathcal{F})$ and Rossby $(\mathcal{R})$ numbers expressed in terms of two independent parameters of the initial flow, namely, the width and the PV of the initial PV strip. The gravity wave flux displays a power dependence on $\mathcal{F}$, up to an optimal $\mathcal{F}$ value, after which the gravity wave flux is inhibited in positive PV trains by background rotation. Rotation introduces a lower cutoff frequency $\omega_{c}=f$ (where $f$ is the Coriolis frequency) in the gravity wave spectra but never prohibits the emission (Ford et al. 2000; Plougonven and Zeitlin 2002). Here we study, among other parameters, the relation of the spontaneous IGW generation with $\mathcal{F}, \mathcal{R}$, and background rotation.

In idealized atmospheric jets, Zhang et al. (2001) and Zhang (2004) found a good fit between the residual of the nonlinear balance equation $\left(\Delta_{\text {nbe }}\right)$ and the emission of IGWs. In these cases the IGWs, generated through balance adjustment, propagated transversely to the potential temperature surfaces. The IGWs investigated here propagate parallel to PV isosurfaces, as in Ford (1994), Lane et al. (2004), Viúdez and Dritschel (2006), and Viúdez (2006). The most popular explanation of SAE is based on the interpretation of the LighthillFord radiation term as a source of IGWs (Ford 1994; Ford et al. 2000; Williams et al. 2003). It has been argued, however, that, for the quasigeostrophic (QG) scaling, the imbalance flow is too small to be reproduced by Lighthill-Ford emission (Saujani and Shepherd 2002). Other indicators of IGWs sources, namely, the horizontal divergence, Richardson number, Brown indicator, and the turbulent energy dissipation rate, have been recently tested by Williams et al. (2005) in a rotating two-layer annulus experiment. These results suggested that the Lighthill-Ford radiation indicator had the best fit with respect to the laboratory observations. How these IGWs are spontaneously generated largely depends on the characteristics of the vortical flow. However, the physical mechanism or mechanisms causing the IGW's emission still remain not well understood. Here we suggest that several mechanisms of spontaneous generation of IGWs are possible. A first mechanism might be related to the advection of $\mathrm{PV}$, while a second mechanism might be related to the horizontal convergence of preexisting IGWs.

Specifically, we analyze the IGWs spontaneously radiated during the merging of two anticyclonic vortices over a range of $\mathcal{R}$ and $\mathcal{F}$. A brief description of the numerical model, initialization procedure, and initial conditions are given in section 2. Section 3 describes the time evolution of the total flow during the merging of the two idealized baroclinic anticyclones. In section 4, the IGWs are identified using the vertical velocity and horizontal velocity divergence. These fields have been selected to visualize IGWs because the ratio between unbalanced and balanced vertical velocity is usually larger than the ratio between unbalanced and balanced horizontal velocity in mesoscale flows (Viúdez and Dritschel 2006; Viúdez 2006). Sensitivity of SAE to sev- 
eral vortex parameters is analyzed in section 5 . The balanced and the unbalanced flows are analyzed in section 6 . In section 7 we address the significance of three different indicators of wave radiation using the numerical simulations or theoretical analysis. Finally, conclusions are given in section 8 .

\section{The numerical model and initial conditions}

\section{a. The numerical model}

We use a three-dimensional triply periodic nonhydrostatic numerical model to simulate rotating, inviscid, stratified, and volume-preserving flows under the Boussinesq and $f$-plane approximations (Dritschel and Viúdez 2003), initialized using the PV initialization approach (VD03). Since the model does not impose the hydrostatic balance, the full range of IGW frequencies (i.e., $\omega \in[f, \mathcal{N}]$ ) is possible, including the pure nonhydrostatic buoyancy waves. The model conserves explicitly the potential vorticity $\Pi$ on isopycnals using the contour-advective semi-Lagrangian algorithm (Dritschel and Ambaum 1997). The nondivergent three-dimensional velocity $\mathbf{u}=(u, v, w)$ and the vertical displacement of isopycnals $\mathcal{D}$ are expressed in terms of the vector potential $\boldsymbol{\varphi}=(\varphi, \psi, \phi)$ so that $\mathbf{u}=-f \boldsymbol{\nabla} \times \boldsymbol{\varphi}$ and $\mathcal{D}=-\epsilon^{2} \boldsymbol{\nabla} \cdot \boldsymbol{\varphi}$, where $\epsilon=1 / c=f / N$ is the ratio between the constant Coriolis and the mean BruntVäisälä frequencies, and $\boldsymbol{\nabla}$ is the three-dimensional gradient operator. The vertical displacement $\mathcal{D}$ of isopycnals with respect to the reference density configuration is $\mathcal{D}(\mathbf{x}, t) \equiv z-d(\mathbf{x}, t)$, where $d(\mathbf{x}, t) \equiv$ $\left[\rho(\mathbf{x}, t)-\rho_{0}\right] / \varrho_{Z}$ is the depth, or vertical location, that an isopycnal located at $\mathbf{x}$ at time $t$ has in the reference density configuration defined by $\rho_{0}+\varrho_{z} z$, where $\rho$ is the density, and $\rho_{0}>0$ and $\varrho_{Z}<0$ are given constants. Note that $\mathcal{D}$ is triply periodic, while $d$ is not. Static instability occurs when $\mathcal{D}_{z} \equiv \partial \mathcal{D} / \partial z>1$. The first two prognostic equations are the material rate of change of the dimensionless horizontal ageostrophic vorticity, defined as

$$
\begin{aligned}
\mathcal{A}_{h} & \equiv(\mathcal{A}, \mathcal{B}) \equiv \boldsymbol{\omega}_{h}^{\prime} / f \equiv\left(\boldsymbol{\omega}_{h}-\boldsymbol{\omega}_{h}^{g}\right) / f \\
& =\boldsymbol{\omega}_{h} / f-c^{2} \nabla_{h} \mathcal{D}=\nabla^{2} \boldsymbol{\varphi}_{h},
\end{aligned}
$$

where $\boldsymbol{\omega}_{h}$ and $\boldsymbol{\omega}_{h}^{g}$ are the total and geostrophic horizontal vorticities, respectively. Using the vorticity equation

$$
\frac{d \boldsymbol{\omega}}{d t}=\boldsymbol{\omega} \cdot \boldsymbol{\nabla} \mathbf{u}+f \mathbf{u}_{z}+N^{2} \mathbf{k} \times \nabla_{h} \mathcal{D},
$$

where $d / d t$ denotes the material time derivative and $\mathbf{k}$ is the vertical unit vector, and the mass conservation equation

$$
\frac{d \mathcal{D}}{d t}=w
$$

the material rate of change of $\mathcal{A}_{h}$ is obtained by

$$
\begin{aligned}
\frac{d \mathcal{A}_{h}}{d t}= & -f \mathbf{k} \times \mathcal{A}_{h}+\left(1-c^{2}\right) \nabla w+f^{-1} \boldsymbol{\omega} \cdot \boldsymbol{\nabla} \mathbf{u}_{h} \\
& +c^{2} \nabla_{h} \mathbf{u} \cdot \boldsymbol{\nabla} \mathcal{D} .
\end{aligned}
$$

The third prognostic equation is simply the explicit material conservation of the PV anomaly $\varpi$ on isopycnals, that is,

$$
\frac{d \varpi}{d t}=0
$$

where the dimensionless PV anomaly is

$\boldsymbol{\varpi} \equiv \Pi-1=\frac{1}{f}(\boldsymbol{\omega}+f \mathbf{k}) \cdot \boldsymbol{\nabla} d=\frac{\zeta}{f}-\mathcal{D}_{z}-\frac{\boldsymbol{\omega}}{f} \cdot \boldsymbol{\nabla} \mathcal{D}$,

and where $\zeta$ is the vertical vorticity. The direct inversion of the Laplace operator, symbolically $\boldsymbol{\varphi}_{h}=\boldsymbol{\nabla}^{-2} \mathcal{A}_{h}$, is used to recover the horizontal components of the vector potential $\boldsymbol{\varphi}_{h}=(\varphi, \psi)$ every time step, while $\phi$, the vertical component of $\boldsymbol{\varphi}$, is obtained from the inversion of the definition of $\varpi$ in (4).

\section{b. Numerical parameters}

Unless otherwise specified we use a $128^{3}$ grid with 128 isopycnals in a domain of extent $L_{X} \times L_{Y} \times L_{Z}$, and ratios $L_{X} / L_{Z}=L_{Y} / L_{Z}$ with $L_{Z}=2 \pi$ (which defines the unit of length). The (mean) buoyancy period $\left(T_{\mathrm{bp}}\right)$ is taken as the unit of time by setting $N \equiv 2 \pi$. One inertial period $T_{\text {ip }}=(N / f) T_{\mathrm{bp}}$. The effect of the stratification versus rotation in the spontaneous-adjustment emission is addressed using two different values of the ratio $c \equiv N / f=L_{X} / L_{Z}=L_{Y} / L_{Z}$ (i.e., quasigeostrophic scaling), namely, $c=10$ and $c=10^{2}$. The time step $\delta t=$ $0.01 T_{\text {bp }}($ case $N / f=10)$, and $\delta t=0.1 T_{\text {bp }}($ case $N / f=$ $10^{2}$ ). Initialization time $\Delta t_{\mathrm{I}}=5 T_{\mathrm{ip}}$. The total period of simulation is $t_{F}=20 T_{\mathrm{ip}}$. The Rossby and Froude numbers are defined as $\mathcal{R} \equiv \zeta / f$ and $\mathcal{F} \equiv\left|\boldsymbol{\omega}_{h}\right| / \mathcal{N}$, where $\mathcal{N}$ is the total Brunt-Väisälä frequency.

\section{c. Initial conditions}

We analyze the emission of IGWs in a range of $R$ and $\mathcal{F}$ in anticyclonic background flow, so chosen because vortices with negative $\varpi$ exhibit larger IGW generation than with positive $\varpi$ (Ford 1994; Viúdez and Dritschel 2006). An isolated anticyclone of minimum PV anomaly, $\varpi_{0}^{-}=-0.95$, exhibits typical $\mathcal{R}_{\min } \simeq-0.90$ and $\mathcal{F}_{\max } \simeq 0.45$. To reach higher $\mathcal{F}_{\max }$ an equally intense $\left(\varpi_{0}^{-}=-0.95\right)$ cooperating anticyclone is added 
TABLE 1. Parameters of the numerical simulations. Initial minimum PV anomaly $\left(\varpi_{0}^{-}\right)$, vortex vertical semiaxis $\left(a_{Z}\right)$, minimum Rossby number $\left(\mathcal{R}_{\min }\right)$, maximum Froude number $\left(\mathcal{F}_{\max }\right)$, and maximum vertical derivative of $\mathcal{D}\left(\mathcal{D}_{z_{\text {max }}}\right)$, for $(i) N / f=10$ and (ii) $N / f=10^{2}$.

\begin{tabular}{lccccc}
\hline \hline Case & $\varpi_{0}^{-}$ & $a_{Z}$ & $\mathcal{R}_{\min }$ & $\mathcal{F}_{\max }$ & $\mathcal{D}_{z_{\max }}$ \\
\hline$i$ & & & $N / f=10$ & & \\
$A_{i}$ & -0.75 & $1 / 4$ & -0.578 & 0.370 & 0.480 \\
$B_{i}$ & -0.85 & $1 / 4$ & -0.708 & 0.492 & 0.562 \\
$C_{i}$ & -0.95 & $1 / 4$ & -0.881 & 0.647 & 0.668 \\
$D_{i}$ & -0.75 & $1 / 2$ & -0.679 & 0.384 & 0.394 \\
$E_{i}$ & -0.85 & $1 / 2$ & -0.773 & 0.501 & 0.466 \\
$F_{i}$ & -0.95 & $1 / 2$ & -0.927 & 0.674 & 0.515 \\
$G_{i}$ & -0.75 & 1 & -0.710 & 0.387 & 0.360 \\
$H_{i}$ & -0.85 & 1 & -0.827 & 0.467 & 0.419 \\
$I_{i}$ & -0.95 & 1 & -0.952 & 0.607 & 0.477 \\
$i i$ & & & $N / f=10^{2}$ & & \\
$A_{i i}$ & -0.75 & $1 / 4$ & -0.578 & 0.370 & 0.480 \\
$B_{i i}$ & -0.85 & $1 / 4$ & -0.709 & 0.491 & 0.562 \\
$C_{i i}$ & -0.95 & $1 / 4$ & -0.879 & 0.648 & 0.669 \\
$D_{i i}$ & -0.75 & $1 / 2$ & -0.658 & 0.396 & 0.404 \\
$E_{i i}$ & -0.85 & $1 / 2$ & -0.774 & 0.501 & 0.467 \\
$F_{i i}$ & -0.95 & $1 / 2$ & -0.928 & 0.675 & 0.516 \\
$G_{i i}$ & -0.75 & 1 & -0.709 & 0.387 & 0.360 \\
$H_{i i}$ & -0.85 & 1 & -0.828 & 0.467 & 0.419 \\
$I_{i i}$ & -0.95 & 1 & -0.959 & 0.608 & 0.477 \\
\hline
\end{tabular}

south of the first one, so that typically $\mathcal{R}_{\min } \simeq-0.92$ and $\mathcal{F}_{\max } \simeq 0.67$ during the merging process.

In the initial configuration the anticyclones are PV ellipsoids. The number of initial PV contours (PV jumps $)$ in the middle isopycnal $\left(i_{\ell}=64\right)$ is $n_{c}=20$, with $\varpi$ varying from $\varpi \cong 0$ on the outermost surface to extreme PV anomaly $\varpi=\varpi_{0}^{-}$at the anticyclone cores. The $\varpi$ increment across each PV jump is fixed for all contours $\Delta \varpi=\varpi_{0}^{-} / n_{c}$ (the exception is the outermost contour where $\left.\Delta \varpi_{0}^{-} / 2\right)$. We consider PV ellipsoids with three different initial configurations, defined such that the outermost PV ellipsoidal layer has ratios between the horizontal $\left(a_{M}=a_{m}=1 / 2\right)$ and vertical $\left(a_{Z}\right)$ semiaxes of $1 / 2,1$, and 2 .

In summary, a set of 18 numerical simulations are analyzed depending on the values of $c \equiv N / f$ (2 values), initial minimum $\mathrm{PV}$ anomaly $\varpi_{0}^{-}$(3 values), and the vortex vertical extent $a_{Z}$ (3 values). Table 1 summarizes the different cases as a function of these parameters.

\section{Merging of two anticyclones}

The PV of the total flow in the reference simulation $F_{i}$ is shown in Fig. 1. The flow is highly ageostrophic and would correspond to two very intense mesoscale ocean anticyclones, with a time average of the minimum $\mathcal{R}$ close to, but smaller than one $\left(\left\langle\mathcal{R}_{\min }\right\rangle=0.91 \pm\right.$ $0.01),\left\langle\mathcal{F}_{\max }\right\rangle=0.57 \pm 0.04$, and is statically stable with

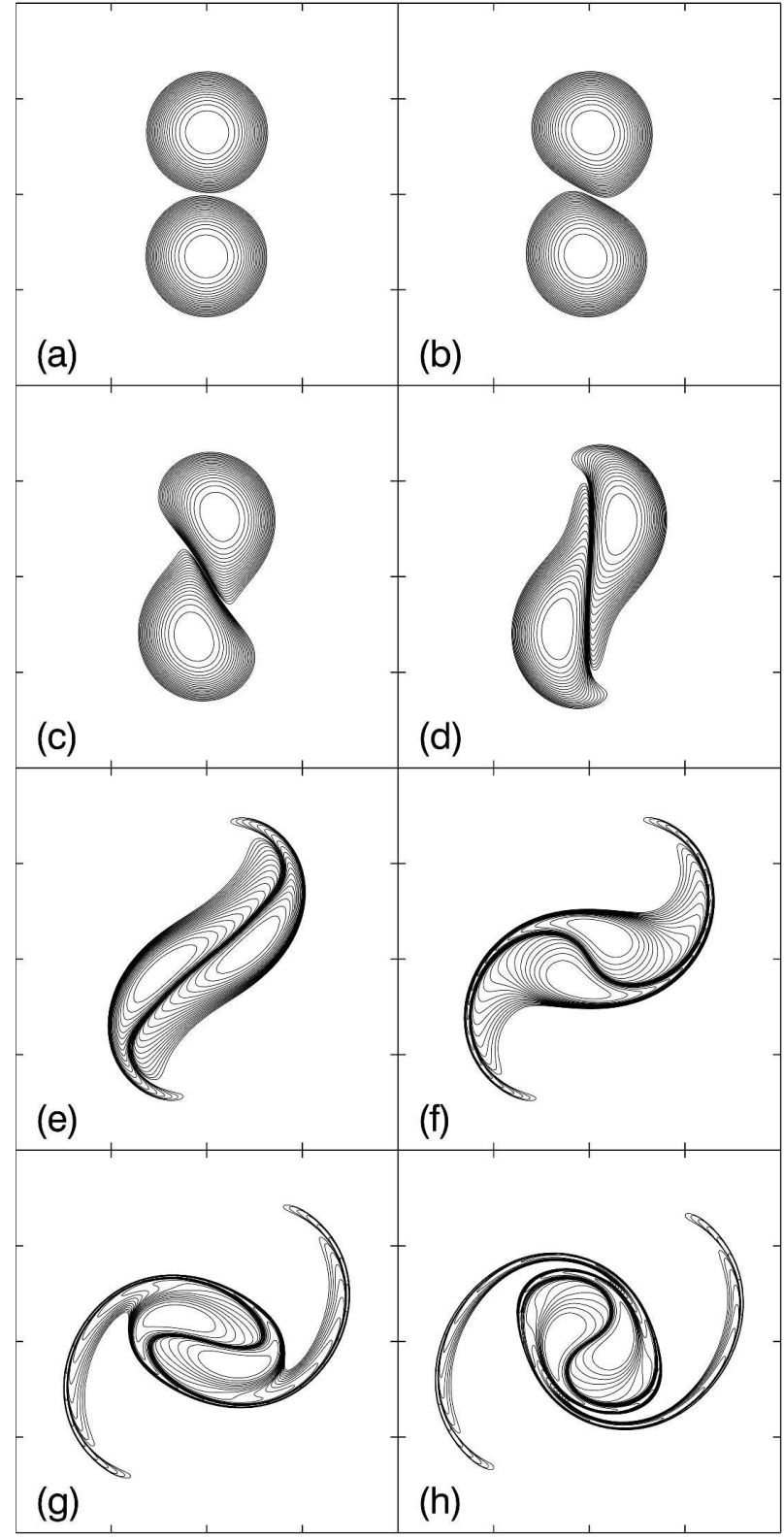

Fig. 1. (a)-(h) $\varpi$ contours from $t=1$ to $8 T_{\text {ip }}$ in the middle isopycnal $\left(i_{\ell}=65, z=0\right)$ for the reference flow $F_{i}$. The complete horizontal domain is shown with $x, y \in[-\pi, \pi] c$.

$\left\langle\mathcal{D}_{z_{\max }}\right\rangle=0.48 \pm 0.03$. The flow is largely in hydrostatic balance. The time average of the ratio between the maximum vertical acceleration $|d w / d t|_{\max }$ and the buoyancy force $N^{2}|\mathcal{D}|$ is

$$
\left\langle\frac{|d w / d t|_{\max }}{\left\{N^{2}|\mathcal{D}|\right\}}\right\rangle_{t} \simeq 2 \times 10^{-3} \ll 1,
$$

where $\left\{N^{2}|\mathcal{D}|\right\}$ is $N^{2}|\mathcal{D}|$ at the location where $|d w / d t|$ is maximum. The corotating vortices interact and merge 

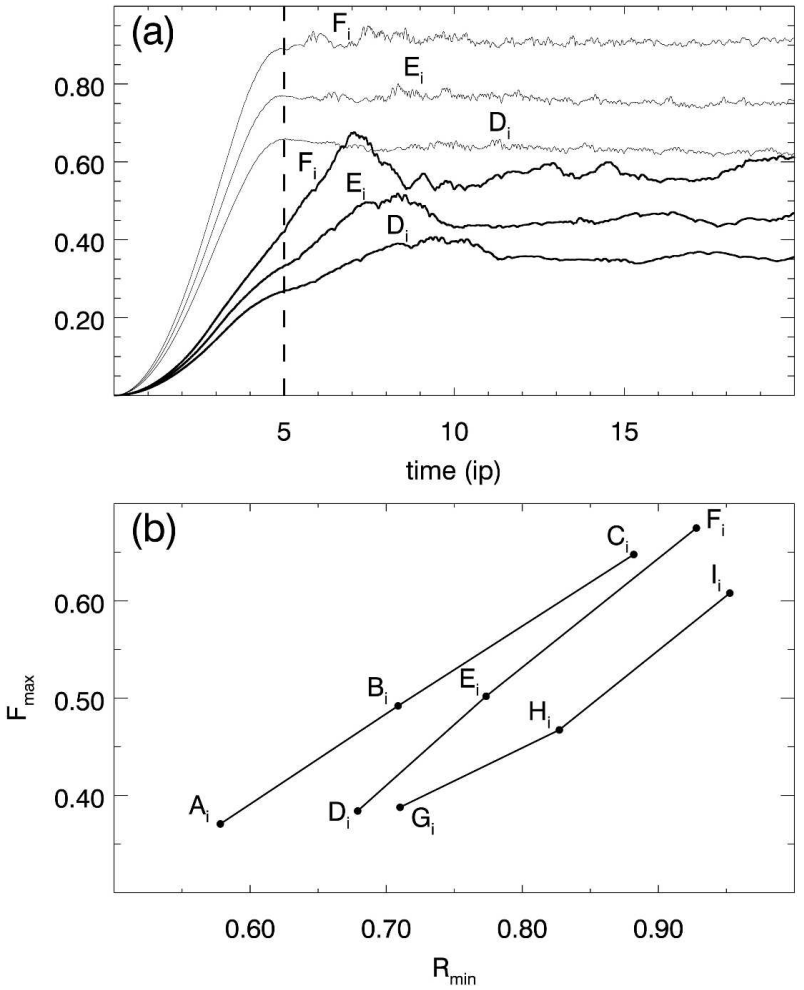

FIG. 2. (a) Time series of $\mathcal{F}_{\max }$ (thick, lower lines) and $\left|\mathcal{R}_{\min }\right|$ (thin, upper lines) for cases $D_{i}-F_{i}$. Time is shown in $T_{\mathrm{ip}}$. (b) $\left|\mathcal{R}_{\min }\right|$ vs $\mathcal{F}_{\max }$ plot for cases $A_{i}-I_{i}$. Parameters for flows $A_{i i}-I_{i i}$ are very similar to these and are not shown.

into a single, bigger, and faster anticyclone. During the vortex merging, from $t=4$ to $4.5 T_{\mathrm{ip}}$, the flow strongly accelerates reaching $\mathcal{R}_{\min }=-0.92$ after initialization (Fig. 2a), while $\mathcal{F}_{\text {max }}$ reaches a characteristic peak $\mathcal{F}_{\max }=0.67$ at $t=7 T_{\text {ip }}$, which is large enough to spontaneously generate IGW packets (Ford 1994; Viúdez and Dritschel 2006; Viúdez 2006). The time series of $\mathcal{R}_{\min }$ and $\mathcal{F}_{\max }$ in Fig. 2a are noisier after $t=5 T_{\text {ip }}$ because of the generation of IGWs (explained below). During and after the vortex merging the resulting elliptical anticyclone filaments symmetrically (Fig. 1, $t=$ $5,6,7$, and $8 T_{\mathrm{ip}}$ ), as is typical in elliptical anticyclones; meanwhile, its core transforms toward a spherical vortex. Anticyclones with smaller $\left|\varpi_{0}^{-}\right|$(cases $D_{i}$ and $E_{i}$ ) reach lower values of $\left|\mathcal{R}_{\min }\right|$ and $\mathcal{F}_{\max }$ (Fig. 2a). As a consequence of the progressively weaker $\left|\varpi_{0}^{-}\right|$the vortical flow is slower, and therefore these three cases $\left(F_{i}\right.$, $D_{i}$, and $E_{i}$ ) differ also in that $\mathcal{F}_{\max }$ peaks at different times.

The scatterplot of parameters $\left|\mathcal{R}_{\min }\right|$ versus $\mathcal{F}_{\max }$ (Fig. $2 b)$ shows that

(i) vortices of equal size (cases $D_{i}-F_{i}$ ) have larger $\mathcal{F}_{\max }$ with respect to shallower (cases $A_{i}-C_{i}$ ) and deeper vortices (cases $G_{i}-I_{i}$ ); (ii) $\left|\mathcal{R}_{\min }\right|$ is larger for deeper vortices (cases $A_{i}-C_{i}$ have $a_{Z}=1 / 4$ while cases $G_{i}-I_{i}$ have $a_{Z}=1$ ); and

(iii) $\mathcal{F}_{\max }$ and $\left|\mathcal{R}_{\min }\right|$ always increase when $\left|\varpi_{0}^{-}\right|$increases.

The differences between the time series of $\left|\mathcal{R}_{\min }\right|$ and $\mathcal{F}_{\max }$ for cases with $N / f=100$ (labeled with subscript "ii" in Table 1) do not show, by inspection, significant changes in tendency and magnitude relative to those in cases with $N / f=10$. In all the simulations $\mathcal{F}_{\text {max }}$, or the inverse square root of the Richardson number (Ri), is smaller than the critical value $\mathcal{F}<\mathcal{F}_{\text {cr }}=2$ (or $\mathrm{Ri}>$ $\left.\mathrm{Ri}_{\mathrm{cr}}=1 / 4\right)$, so that Kelvin-Helmholtz instability does not develop.

The spontaneous-adjustment emission is not appreciated in Fig. 1 because the IGWs have relatively small horizontal velocity and approximately zero PV. The IGWs are, however, easily visualized (next section) in the vertical velocity field $\left[O(w) \sim 10^{-5}\right]$ or in its vertical derivative, that is, the (minus) horizontal divergence, $\boldsymbol{\nabla}_{h} \cdot \mathbf{u}_{h}=-\partial w / \partial z$.

\section{The spontaneous-adjustment emission of inertia-gravity waves}

\section{a. Mesoscale vertical velocity}

The evolution of the mesoscale vertical velocity of the total flow is shown in Fig. 3. The pattern of $w$ displays the typical octupole in the three-dimensional space, or quadrupole in the horizontal plane, of alternating positive-negative cells (VD03). Extreme values of $w$, located close to the edges of the PV ellipsoid, reach $10 \times 10^{-5}, 8 \times 10^{-5}$, and $7 \times 10^{-5}$ at $t=5,6$, and $7 T_{\text {ip, }}$, respectively.

Short-scale IGWs are identifiable as perturbations in the otherwise quadrupolar pattern of $w$ contours (initially in the northeast and southwest sides of the vortex at $t=6$ and $7 T_{\mathrm{ip}}$ ), and also on the vertical distribution of $w$ at $z \leq \pi / 2$ (Fig. 4). Because of the large $\left|\mathcal{R}_{\min }\right|$ and $\mathcal{F}_{\max }$ of the reference flow $F_{i}$, the vortex starts spontaneously emitting IGWs of smaller amplitude before the end of the initialization time $\Delta t_{I}=5 T_{\text {ip }}$ (slightly noticeable in Fig. 3a2). To avoid the large vortex mesoscale $w$, the horizontal distributions in Fig. 3 correspond to a deep layer $\left(z \simeq-1.40\right.$, i.e., deeper than $\left.a_{Z}=1 / 2\right)$, so that the waves observed in this layer have been generated in the middle isopycnal $(z=0)$ at $t<5 T_{\mathrm{ip}}$. The IGWs, however, acquire larger amplitude after $t_{I}=5$ $T_{\mathrm{ip}}$, rapidly spreading and dispersing away from the wave sources. This particular spontaneous generation is a vortex-wave interaction in the sense that it is reproduced by the full dynamical equations, and not by any balanced and wave dynamics separately.

The IGWs propagate horizontally eastward and 


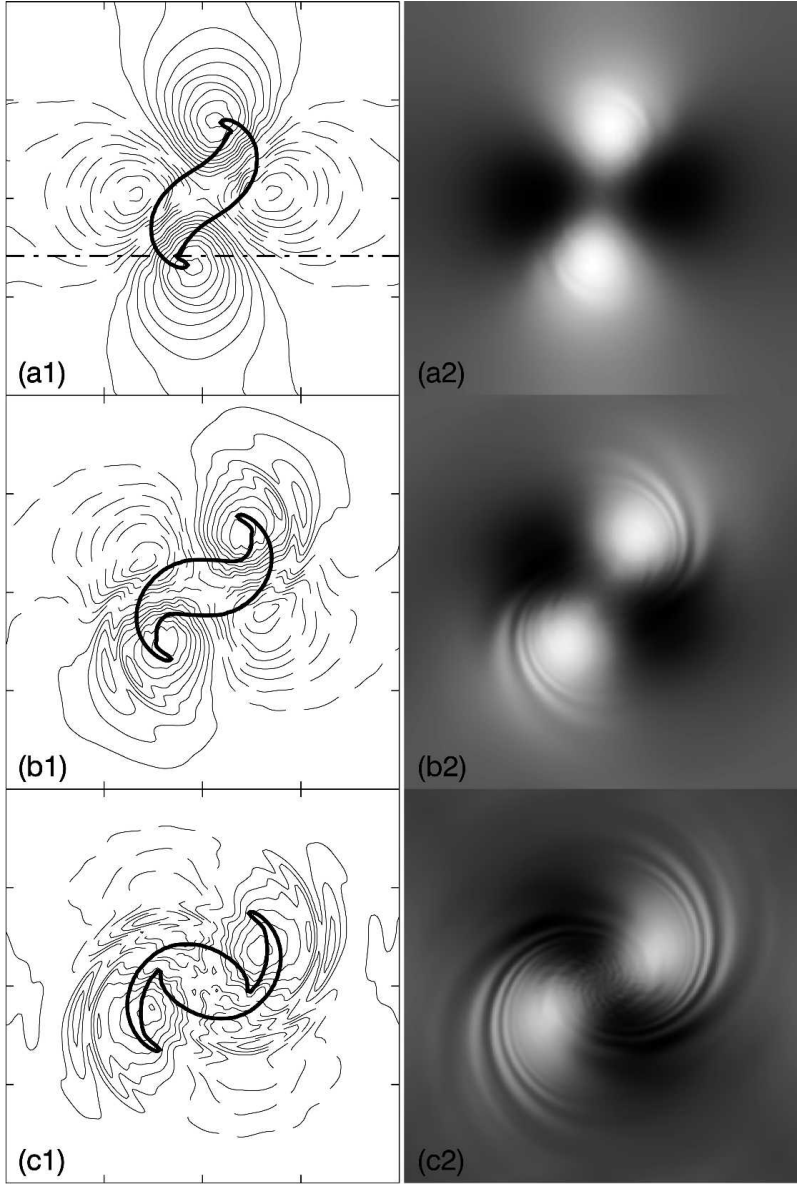

FIG. 3. Horizontal distributions of $w$ at (a) $t=5 T_{\text {ip }}(w \in[-9.2$, $\left.10.4] \times 10^{-5}\right)$, (b) $6 T_{\text {ip }}\left(w \in[-8.8,8.9] \times 10^{-5}\right)$, and (c) $7 T_{\text {ip }}$ $\left(w \in[-6.9,7.2] \times 10^{-5}\right)$ on the plane $i_{Z}=36(z \simeq-1.42)$ for the reference flow $F_{i}$ (with a $128^{3}$ grid), shown as contour plots (script 1 , dashed contours mean negative values, and contour interval $\Delta w=10^{-5}$ ), and as images (script 2 , with $256^{2}$ pixels, in a common black and white scale using extrema $\left.w \in[-9.2,10.4] \times 10^{-5}\right)$. The PV contour $\varpi=-0.01$ (thick solid line) and the vertical section [thin dashed line in (a1)] are included for reference. Horizontal domain is $x, y \in[-\pi, \pi] c$.

westward as symmetric wave packets, owing to the initial vortex symmetry (Figs. 3b,c), and vertically downward and upward (Fig. 4). We note that the spiral IGW packets propagate in the three-dimensional space, with positive phase velocity and negative group velocity in the lower half of the domain (the opposite happens in the upper half). As a consequence of the vortex filamentation and loss of sphericity the amplitude of the balanced larger-scale $w$ decreases with time (cf. the extrema of the quadrupole pattern of $w$ in Figs. 3b-d).

The wave fronts of the wave packet display a spiral pattern (Figs. 3b2, 3c2), which is a characteristic property of waves radiated from a rotating source. The sense of spiraling, as one moves from the older crests located

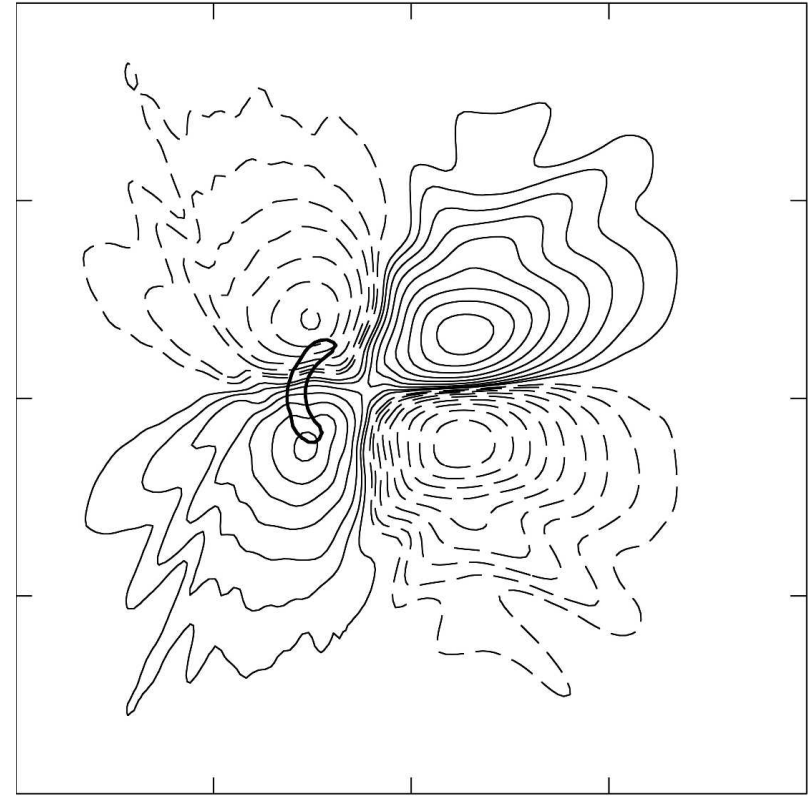

FIG. 4. Vertical distribution of $w$ at $t=7 T_{\text {ip }}(w \in[-4.8,4.6] \times$ $\left.10^{-4}\right)$ on the plane $i_{Y}=46(y \simeq-0.93 c)$ for the reference flow $F_{i}$ (contour levels equal to $\{\ldots,-4,-3,3,4, \ldots\}^{2.5} \times 10^{-6}$ ). The PV contour $\varpi=-0.01$ (thick solid line) is included for reference. Domain is $x / c, z \in[-\pi, \pi]$.

away from the vortex to the new crests located close to the vortex, is clockwise; this is the same sense as the phase rotation of the anticyclone. The wavelength of the IGWs is shorter close to the vortex edge, where the local rate of change of PV is larger, and increases as the wave packet propagates away. The typical horizontal and vertical wavelengths $\left(\lambda_{h}\right.$ and $\left.\lambda_{v}\right)$ of the IGWs are $O\left(\lambda_{h} / c\right) \sim O\left(\lambda_{v}\right) \sim 1$, respectively. The grid spacing $\Delta=$ $2 \pi / 128$ is small enough to resolve most of the IGWs generated spontaneously. Note also in Fig. 5b the modification of the spiral crests by the strong PV gradients in the north and south edges of the PV vortex (Fig. 5a). The three-dimensional spiral IGW packets originate on the middle isopycnal, with two symmetrical wave packets propagating in the upper $(z>0)$ and lower $(z<0)$ layers (Fig. 4).

A new SAE event occurs in the vortex interior at $t \simeq$ $7 T_{\mathrm{ip}}$, when two symmetric wave packets are generated (Fig. 5b). Quite surprisingly, this second event is stronger than the first one. These IGWs do not spread away as fast as the ones in the first event, but seem to remain longer trapped inside the vortex, with their wave fronts forming — rather than a spiral—an annular pattern.

\section{b. Horizontal divergence}

The horizontal divergence of the horizontal velocity $\boldsymbol{\nabla}_{h} \cdot \mathbf{u}_{h}$ (hereinafter, just divergence) displays a pattern 


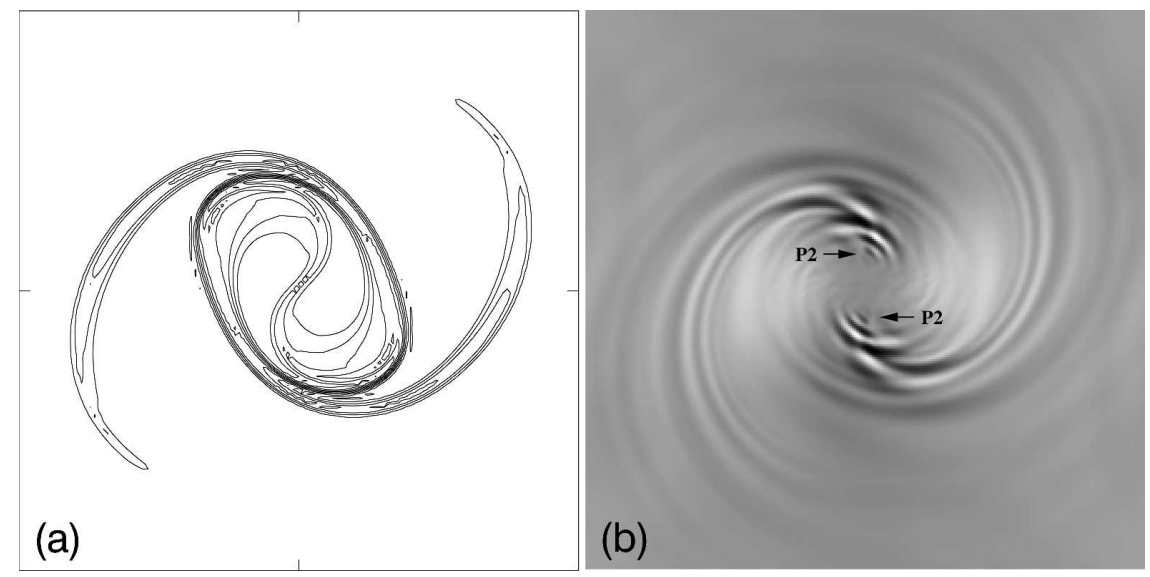

FIG. 5. Horizontal distributions of (a) $\varpi$ at $z=0$ (contour interval $\delta \varpi=0.15$, minimum contour $\left.\pm 5 \times 10^{-2}\right)$, and (b) $w$ at $z \simeq-1.42\left(w \in[-19.4,9.7] \times 10^{-5}\right)$, for the reference case $F_{i}$ at $t=8 T_{\mathrm{ip}}$. Domain is $x, y \in[-\pi,+\pi] c$.

similar to the $w$ field, both having the same sign in the lower half of the domain (Fig. 6). This is because the flow is volume-preserving (nondivergent) and $w=0$ in the middle, bottom, and top layers. Thus, $\boldsymbol{\nabla}_{h} \cdot \mathbf{u}_{h}>0$ indicates upwelling in the lower layers, while the opposite happens in the upper layers. Since the IGWs have a spatial scale shorter than the balanced flow, their spiral signature appears more clear in the divergence field $\left(-w_{z}\right.$, Fig. 6b) than in the total $w$ field (Fig. 3c2).

\section{c. Numerical resolution}

The reference case $F_{i}$ was repeated with double resolution $\left(256^{3}\right.$ grid points) to verify the convergence of the main results to increasing numerical resolution. Figure 7 shows the resulting horizontal and vertical distributions of $w$. The generation of spiral IGWs is well reproduced in the $256^{3}$ case, with the wave phases at the same location and time relative to those in the $128^{3}$ case (Figs. 3b1, 3c1, and 4). The horizontal and vertical wavelengths are well preserved at $256^{3}$, though as expected, significant changes in the PV gradients and vertical velocity shears are generated at the smaller scales (not shown). As a consequence of the higher resolution the extreme values of $w$ are enhanced by an amount $\Delta w \simeq 10^{-5}$, and some new relative $w$ extrema appear (Fig. 7).

The time averages of the wavenumber spectra of $w$, $\left\langle\hat{w}^{2}\right\rangle_{t}(\kappa)$, for the reference case $F_{i}$ with $64^{3}, 128^{3}$, and $256^{3}$ grid points (Fig. 8a) show that the change of numerical resolution has little effect for low wavenumbers $(\kappa<4)$, while the numerical simulations with increasing resolution resolve better the smaller scales so that more

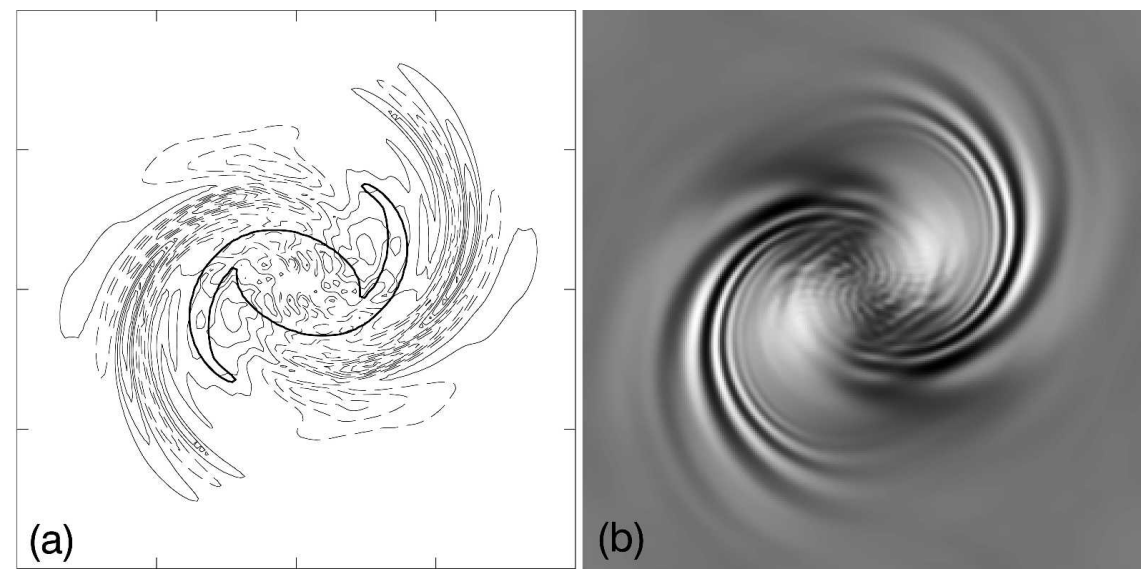

FIG. 6. Horizontal distributions of $\boldsymbol{\nabla}_{h} \cdot \mathbf{u}_{h}=-w_{z}$ at $t=7 T_{\text {ip }}\left(-w_{z} \in[-2.9,2.0] \times 10^{-4}\right)$ on the plane $z \simeq-1.42$ for the reference flow $F_{i}$ (with a $128^{3}$ grid), as a contour plot $\left[\Delta\left(-w_{z}\right)=\right.$ $\left.4 \times 10^{-5}\right]$, and as an image (with $256^{2}$ pixels, black and white scale with $-w_{z} \in[-2.9,2.0] \times$ $10^{-4}$ ). The PV contour $\varpi=-0.01$ (thick solid line) is included. Domain is $x, y \in[-\pi, \pi] c$. 


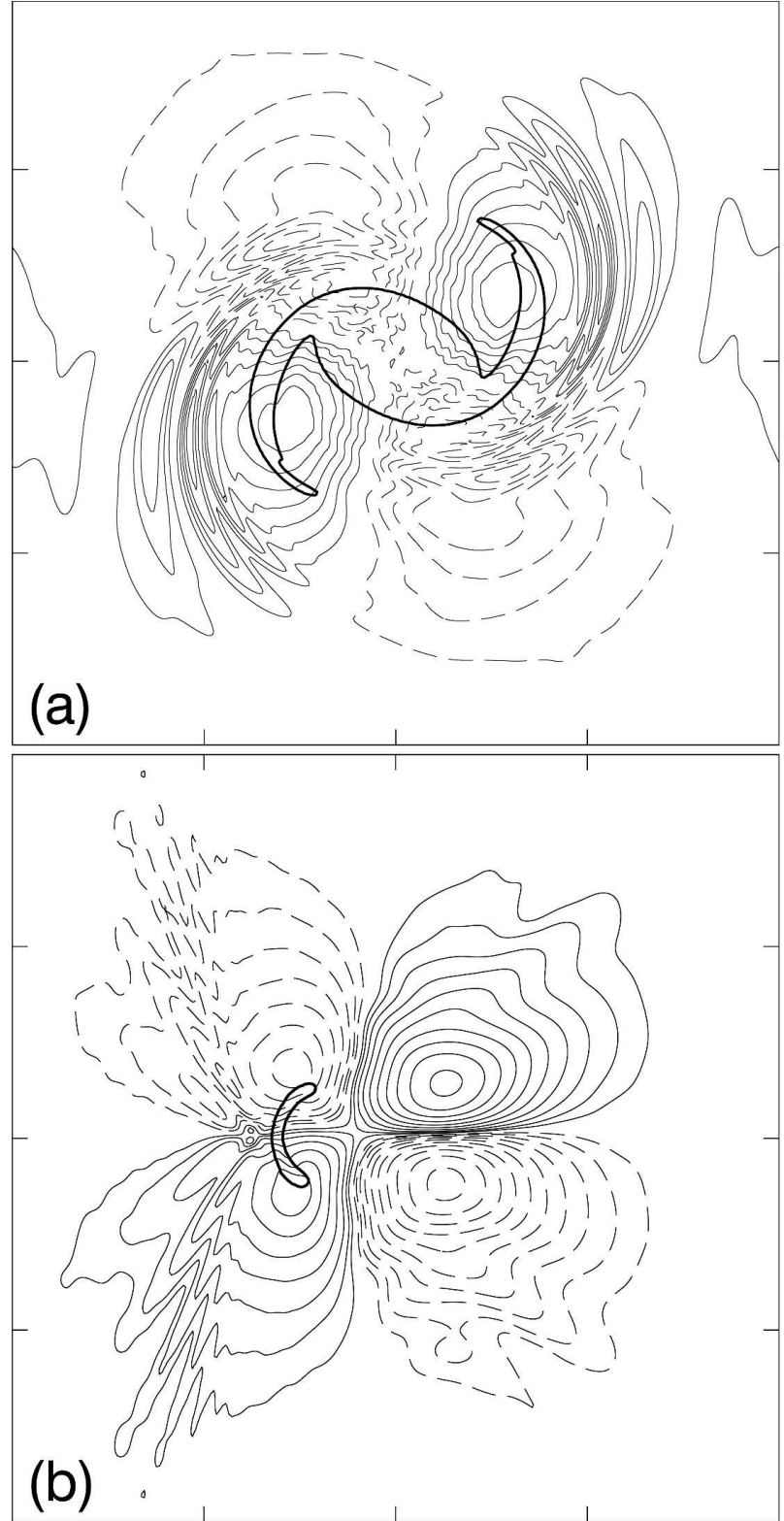

FIG. 7. (a) Horizontal $(z \simeq-1.42)$ and (b) vertical $(y \simeq-0.93 c)$ distributions of $w$ at $t=7 T_{\text {ip }}$ for the reference case $F_{i}$ with high numerical resolution ( $256^{3}$ grid points). Contour interval and line styles are as in Figs. 3 and 4. The PV contour $\varpi=-0.01$ (thickest solid line) is included.

kinetic energy of the vertical motion is transferred from the middle $(4<\kappa<40)$ to the high wavenumbers $(\kappa>$ $40)$. This is consistent with the intensity enhancement of the small-scale IGWs observed in the $w$ field (Fig. 7). The slope of the spectra of the high-resolution simulations are flatter between $\kappa \simeq 10$ and 40 , indicating the generation of IGWs that are not well resolved at $64^{3}$ resolution. The energy of these unresolved small-scale waves is shifted to the larger scales in the low-resolu- tion case (Fig. 8a). The energy transfer from low to high wavenumbers during the spontaneous generation of IGWs in the high-resolution case is shown in the time evolution of the wavenumber spectra (Fig. 8b).

\section{d. Numerical diffusion}

The numerical diffusion needed to avoid the growing of grid-size noise, consists of a hyperdiffusion term in the equations for the rate of change of $\mathcal{A}_{h}$, that is, $-\mu\left(\nabla_{q}^{4} \mathcal{A}, \nabla_{q}^{4} \mathcal{B}\right)$, where $\nabla_{q} \equiv\left(\partial_{x}, \partial_{y}, \epsilon \partial_{z}\right)$ is the gradient operator in the vertically stretched (QG) space. The hyperviscosity coefficient $\mu$ is chosen by specifying the damping rate ( $e$-folding, $e_{f}$ ) of the largest wavenumber in spectral space per inertial period, which was set constant to a nominal value of 50 . The aim is to keep the numerical diffusion large enough to damp the grid-size noise but small enough to allow the spontaneous generation of IGWs. Owing to the small amplitude of the spontaneous IGWs, the sensitivity of the IGWs' generation to the hyperviscosity coefficient has been analyzed, in the reference case $F_{i}$, using an $e$-folding coefficient twice its nominal value. The results show that the $\mathrm{PV}$ of the anticyclone is not appreciably weakened by the higher diffusion, and the generation of IGWs occurs at the same time and location as in the low diffusion case (not shown).

The differences in the magnitude of $w$ are analyzed in terms of the spectra. The three-dimensional spatial average of the frequency spectra of $w,\left\langle\hat{w}^{2}\right\rangle_{x y z}(\omega)$, is shown in Fig. 9a. The IGWs appear as a relative maximum at high frequencies $\omega \simeq 0.15 T_{\mathrm{bp}}^{-1}$. The IGWs are, however, better observed at deeper layers, where the balanced $w$ is smaller, so that the horizontal averages of the frequency spectra of $w,\left\langle\hat{w}^{2}\right\rangle_{x y}(z, \omega)$, were computed as a function of $z$. At $z=-2.36$ (Fig. 9b) $\left\langle\hat{w}^{2}\right\rangle_{x y}$ has an absolute maximum corresponding to the spontaneous IGWs, while the vortex $w$ at this depth is much smaller than in the shallow layers. In both cases $\left(e_{f}=50\right.$ and $\left.e_{f}=100\right)$ the spectra look similar though, as expected, the IGW amplitude decreases for increasing diffusion. Thus, doubling the coefficient of numerical diffusion did not result in an inhibition of SAE, but in a small decrease in the amplitude of the spontaneous IGWs. The local frequency and wavelength (not shown) of the IGWs are not seriously affected by the increase of the diffusion rate.

\section{Sensitivity of SAE to the vortex flow characteristics}

Spontaneous generation of IGWs is a robust phenomenon in balanced mesoscale vortical flows with moderate or large $\mathcal{F}_{\text {max }}$. Unstable baroclinic jet cur- 

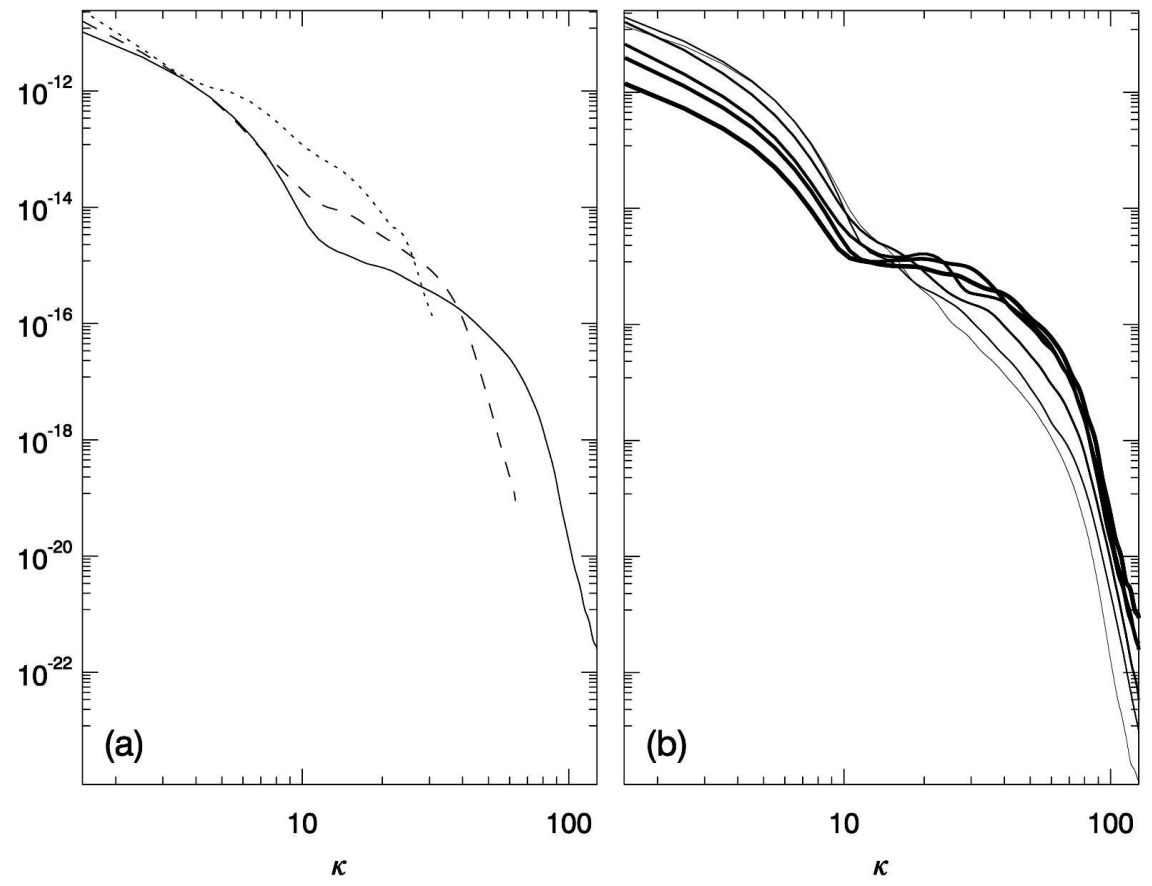

FIG. 8. (a) $\left\langle\hat{w}^{2}\right\rangle_{t}(\kappa)$, the time average (from $t=50$ to $100 T_{\mathrm{bp}}$ every $T_{\mathrm{bp}}$ ) of the wavenumber spectra of $w$ for the reference case $F_{i}$ and numerical resolutions with $64^{3}$ (short dashed), $128^{3}$ (long dashed), and $256^{3}$ (solid) grid points. (b) Wavenumber spectra $\hat{w}^{2}(\kappa, t)$ for the $256^{3}$ case from $t=5$ to $10 T_{\text {ip }}$ (line thickness increases with time). The horizontal axis is the total wavenumber $\kappa=2 \pi\left(k^{2}+l^{2}+m^{2}\right)^{1 / 2}$.

rents, vortices with no radial symmetry, and dipoles are some examples of mesoscale balanced flows that generate spiral patterns of IGWs (Viúdez and Dritschel 2006; Viúdez 2006). The intensity of SAE events as a function of the maximum PV anomaly $\varpi_{0}^{-}$, vortex vertical extent $a_{Z}$, and ratio $N / f$ are described in this section. As explained in the previous section we use the horizontal spatial average, rather than the threedimensional spatial average, of frequency spectra.

\section{a. The effect of $\varpi_{0}^{-}$}

Figure 10 shows the frequency spectra $\left\langle\hat{w}^{2}\right\rangle_{x y}$ for cases with different $\varpi_{0}^{-}\left(D_{i}, E_{i}\right.$, and $\left.F_{i}\right)$. Maximum values happen at low frequencies $\left(\omega<0.02 T_{\mathrm{bp}}^{-1}\right)$ corresponding to the components of the near-balanced flow of the anticyclone, which has a phase rotation period $\tau_{b} \simeq 10 T_{\mathrm{ip}}$ (frequency $\omega_{b}=0.01 T_{\mathrm{ip}}^{-1}$ ). Besides the balanced flow, IGWs are generated in all cases. At deep layers the spectra $(1,2$, and 3$)$ display two jumps at $\omega \simeq$ 0.10 and $1 T_{\mathrm{bp}}^{-1}$ that correspond to the range of the IGW frequencies, that is, $\omega \in[f, N]$. The IGWs are more energetic close to the inertial frequency than to the buoyancy frequency. In shallower layers (spectra 4), the IGWs are largely masked by the mesoscale $w$ and only appear as small fluctuations in the IGW range of frequencies.
An increment of $\left|\varpi_{0}^{-}\right|$in the vortices results in an enhancement of the IGW components; therefore, IGW packets of larger amplitude are generated. Large differences in $\mathcal{F}_{\max }$ between different flows (Fig. 2a) induce large differences in the energy radiated by the balanced flow (Fig. 10). On the other hand, as $\left|\varpi_{0}^{-}\right|$ increases, so does the relative vorticity in the anticyclone, because it is larger than the available energy of the mean flow that is transferred to the imbalanced flow as IGWs. Thus, large $\mathcal{F}_{\max }$ and $\left|\mathcal{R}_{\min }\right|$ favor the spontaneous emission of IGWs.

\section{b. The effect of $a_{Z}$ and $N / f$}

The initial vortices in cases $C_{i}, F_{i}$, and $I_{i}$ (Table 1) have the same $\varpi_{0}^{-}$but different vertical extent $a_{Z}$. As can be observed in Fig. 11, IGWs are always generated, though the spontaneous emission in the shallow vortices (case $C_{i}$ ) is weaker than in the deep vortices (cases $F_{i}$ and $\left.I_{i}\right)$. When $a_{Z}$ is increased the amount of anticyclonic vorticity increases as well, so that IGW packets with larger amplitude are emitted. Differences in $\left\langle\hat{w}^{2}\right\rangle_{x y}$ between cases $F_{i}$ and $C_{i}$ are larger than between $I_{i}$ and $F_{i}$, suggesting that, for static and inertially stable flows, there is a maximum for the rate of change of spontaneous IGW emission. We note that flows $F_{i}$ and $I_{i}$ are close to the limit of inertial stability. 


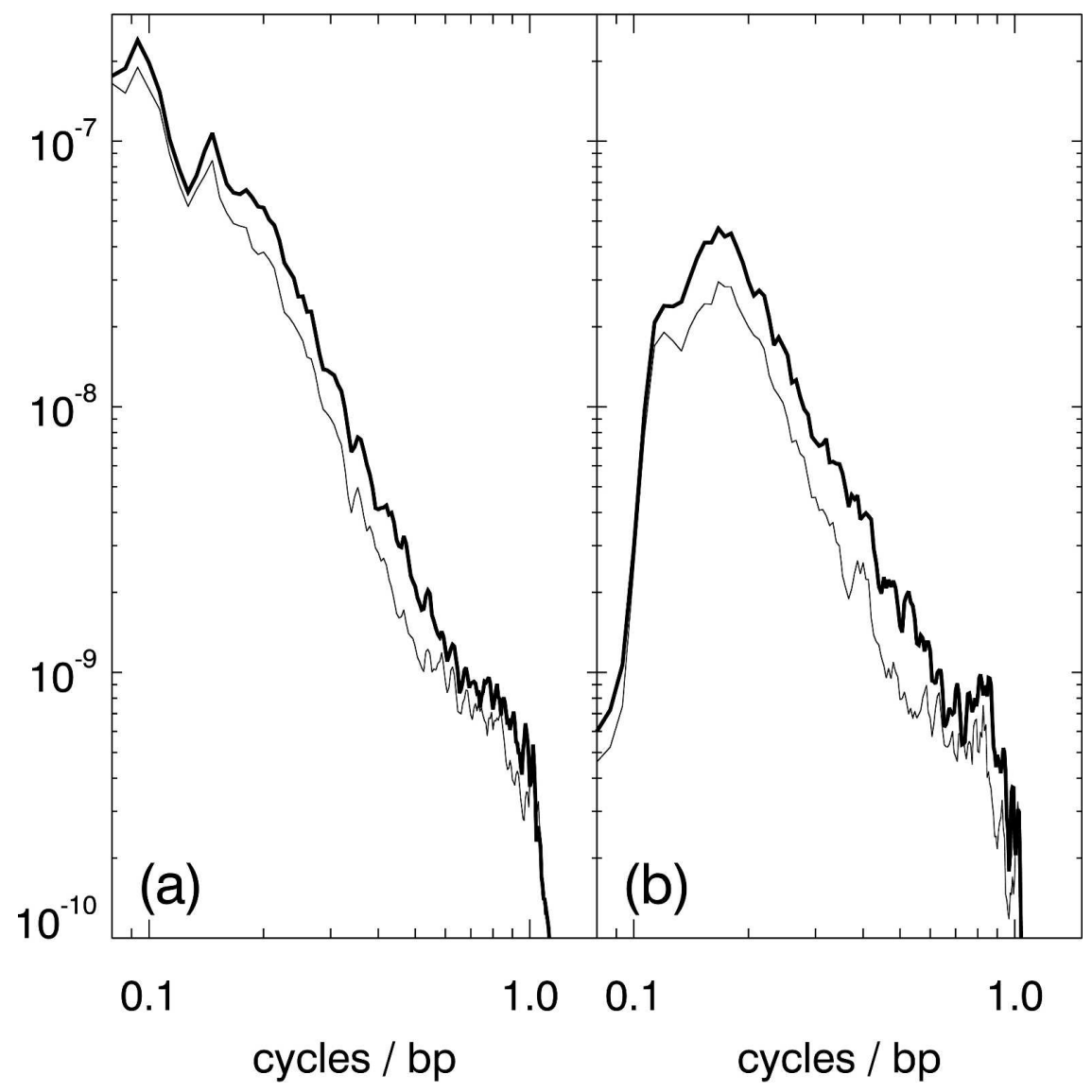

FIG. 9. (a) The frequency spectra $\left\langle\hat{w}^{2}\right\rangle_{x y z}(\omega)$, the three-dimensional spatial average of $\hat{w}^{2}(\mathbf{x}$, $\omega)$, and (b) $\left\langle\hat{w}^{2}\right\rangle_{x y}(z, \omega)$, the horizontal average of the frequency spectra of $w$, at $z \simeq-2.36$, for the reference case $F_{i}$, with $e_{f}=50$ (thick solid line) and $e_{f}=100$ (thin solid line). Every spectrum is the average of (a) $8^{3}$ and (b) $8^{2}$ spectra computed from $t=50$ to $200 T_{\mathrm{bp}}$. The time interval of the time series is $\delta t=10^{-2} T_{\mathrm{bp}}$. Only frequencies in the range [0.08, 1.10] $T_{\mathrm{bp}}^{-1}$ are shown.

An increase of $c \equiv N / f$ from $c=10$ to 100 implies that the range of IGW frequencies increases from $\delta \omega=$ $[0.1,1]$ to $[0.01,1] T_{\mathrm{bp}}^{-1}$. Thus, the spectra of IGWs in the flows with $c=100$ is broader than those with $c=$ 10 (Fig. 12). Also, since the QG scaling $\left(L_{X} / L_{Z}=\right.$ $\left.L_{Y} / L_{Z}=N / f=c\right)$ is always used, the vertical velocity is inhibited for larger $c$, so that the (squared) spectral amplitudes of $w$ in the flows with $c=10^{2}$ are reduced by a factor of $10^{2}$ with respect to the flows with $c=10$ (Fig. 12). We note also that, since the extent of the PV vortices are kept constant in the stretched space, the PV gradients, and hence the PV advection, decrease with increasing $c$.

\section{Balance and imbalance flow}

To analyze the spontaneous emission of IGWs it is very useful to separate, as long as it is possible, the balance and imbalance components of the flow. To do so we have used the optimal potential vorticity (OPV) balance approach (Viúdez and Dritschel 2004b), which extracts the balanced vector potential $\left(\varphi_{b}\right)$ from the potential of the total flow $(\varphi)$. The balanced fields $\varphi_{b}(\mathbf{x}, t)$ were obtained every $T_{\mathrm{bp}}$ from $t=50$ to $t=85$ $T_{\mathrm{bp}}$, and only for the reference case $F_{i}$ since the OPV balance is computationally expensive, especially for flows with large $|R|$.

\section{a. The balanced flow}

The horizontal distributions of the balanced vertical velocity $w_{b}$, obtained from $\boldsymbol{\varphi}_{b}$ (Fig. 13), display the typical quadrupolar pattern of $w$. There are also some waves present in $w_{b}$. These waves are those being generated at the time of diagnostics, which being part of the full dynamics at that time cannot be fully extracted from the OPV balanced potentials. That is, since they are spontaneously generated by the balanced flow they do not obey, at the time of generation, an imbalance dynamics. Thus, the efficiency of the OPV balance di- 


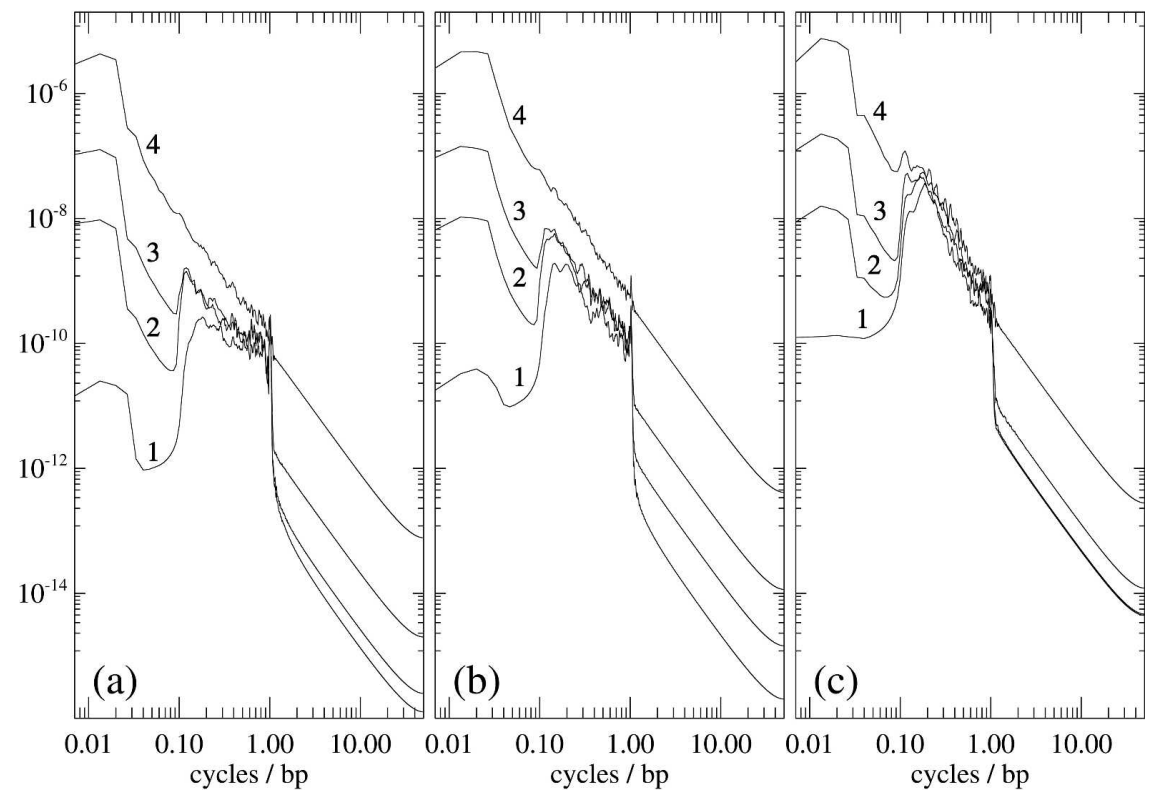

FIG. 10. $\left\langle\hat{w}^{2}\right\rangle_{x y}(z, \omega)$, the horizontally averaged frequency spectra of $w$ at $z=-\pi(1), z \simeq$ -2.36 (2), $z \simeq-1.57$ (3), and $z \simeq-0.79$ (4) for the cases (a) $D_{i}$, (b) $E_{i}$, and (c) $F_{i}$. Every spectrum is the average of $8^{2}$ spectra computed from $t=50$ to $t=200 T_{\mathrm{bp}}$. The time interval of the time series is $\delta t=10^{-2} T_{\mathrm{bp}}$.

agnosis is inversely related to the closeness of the SAE event to the diagnosis time. However, visual comparison between Figs. 3b1, 3c1 and Figs. 13a,b shows that the largest part of the waves are extracted by the OPV balance approach. The magnitude of $w_{b}$ is $O\left(w_{b}\right)=$ $10^{-5}$, the same as $w$ (Figs. 3a1-3c1). The difference between the $w_{b}$ in Figs. 13a,b and Fig. 13c is because the packet observed at $t=8 T_{\text {ip }}$ (Fig. 5b) has been recently generated and has a magnitude larger than the wave packet observed at $t=5 T_{\text {ip }}$ (Fig. 3a).

\section{b. The unbalanced flow}

Once $\boldsymbol{\varphi}_{b}$ is known, the imbalance vector potential $\boldsymbol{\varphi}_{i}$ is extracted from the total flow $\boldsymbol{\varphi}_{i} \equiv \boldsymbol{\varphi}-\boldsymbol{\varphi}_{b}$, and the

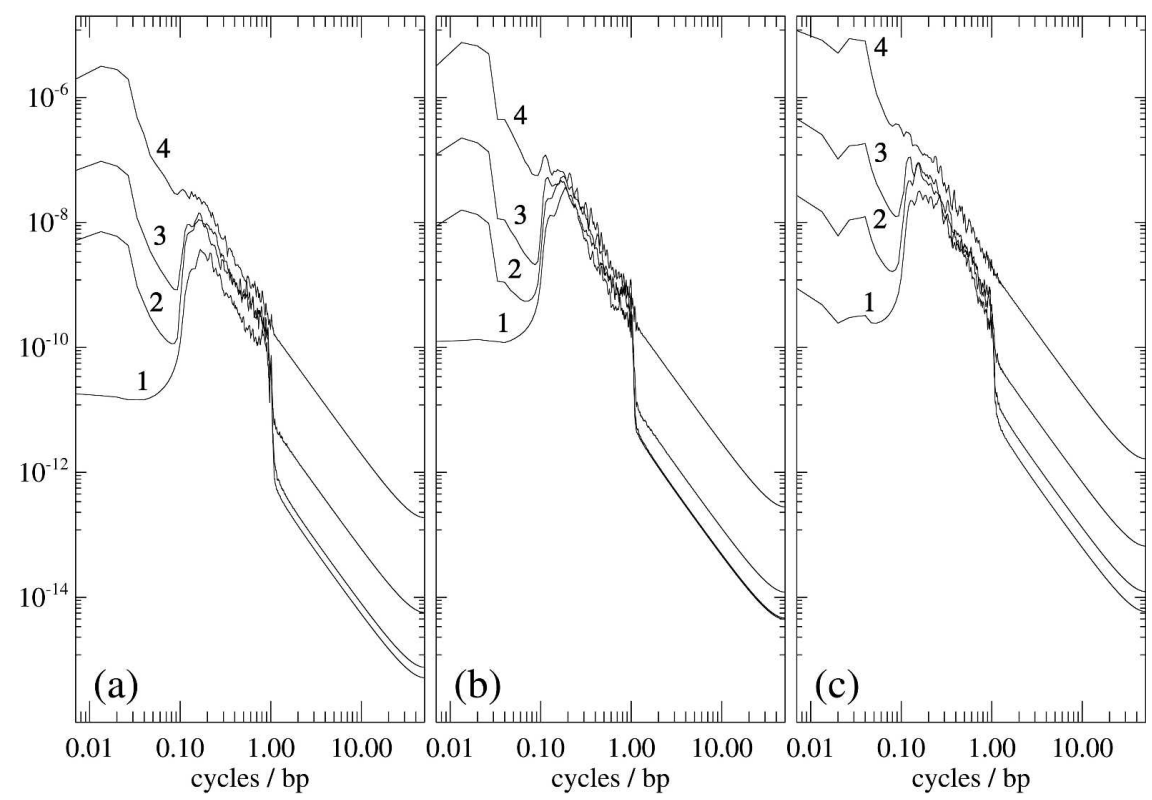

FIG. 11. Same as in Fig. 10 but for the cases (a) $C_{i}$, (b) $F_{i}$, and (c) $I_{i}$. 

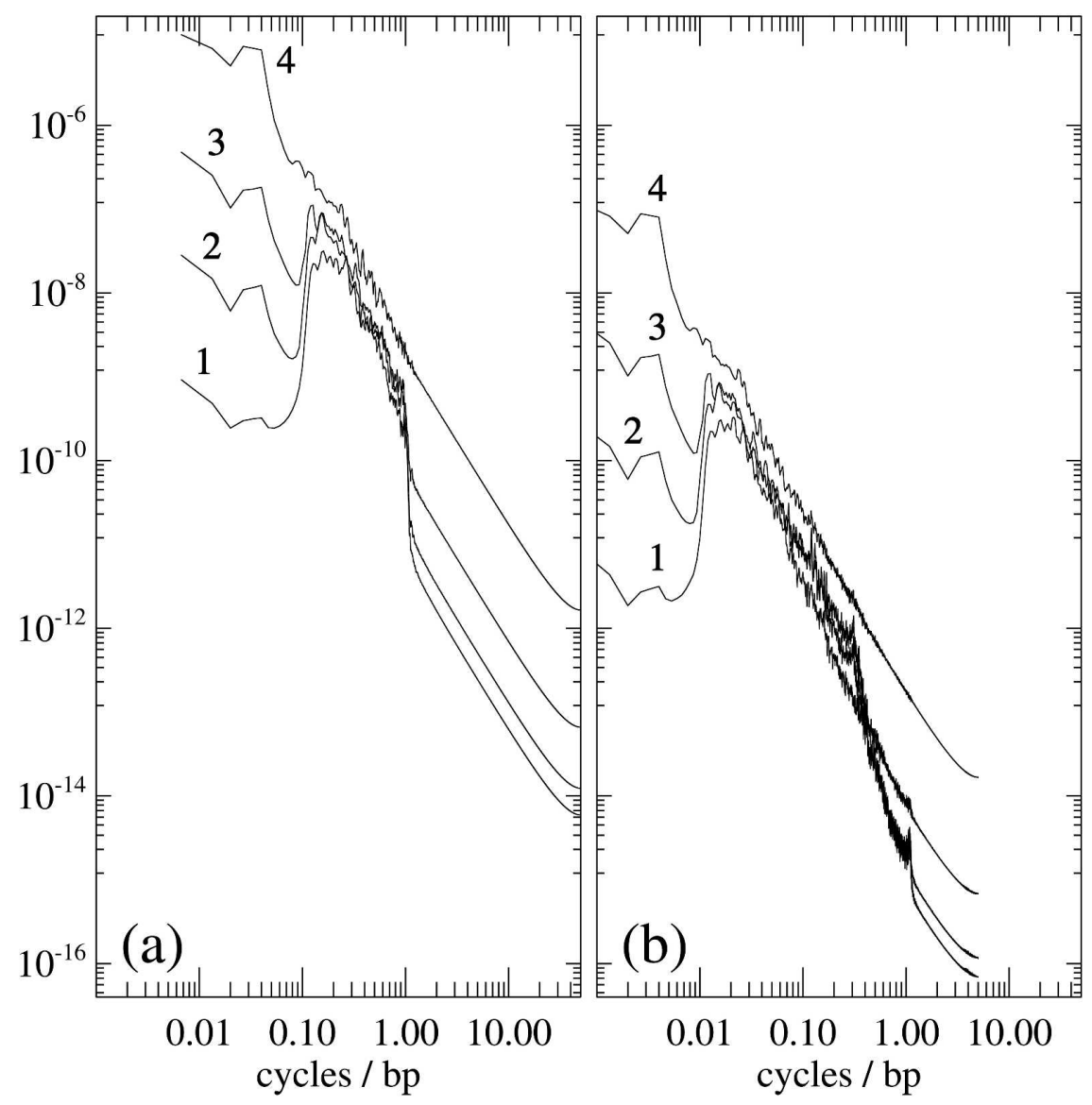

FIG. 12. Same as in Fig. 10 but for the cases (a) $I_{i}$ and (b) $I_{i i}$.

unbalanced quantities can be obtained: $\mathbf{u}_{i} \equiv-f \boldsymbol{\nabla} \times$ $\boldsymbol{\varphi}_{i}=\mathbf{u}-\mathbf{u}_{b}$, and $\mathcal{D}_{i} \equiv-\epsilon^{2} \boldsymbol{\nabla} \cdot \boldsymbol{\varphi}_{\mathrm{i}}=\mathcal{D}-\mathcal{D}_{b}$.

The $w_{\mathrm{i}}$ is one order of magnitude smaller than $w$, $O(w) / O\left(w_{i}\right)=10^{-5} / 10^{-6}=10$. The time evolution of the waves along horizontal and vertical planes is shown in Figs. 14 and 15, respectively. The first IGW packet (hereafter, $\mathrm{P}_{1}$ ) has started propagating away from the vortex at $t=5 T_{\text {ip }}$ (Fig. 14a), which is related to the large PV anomaly $\left(\varpi_{0}^{-}=-0.95\right)$ of the vortices in the reference case. Between $t=5$ and $t=5.5 T_{\text {ip }}$ new smaller-scale and small-amplitude waves (S in Figs. 14c and $15 \mathrm{c}$ ) are generated and propagate from the interior to the vortex edges (Figs. 14b,c and 15b-d). These small-amplitude waves have wavenumber vectors in the same direction as those of the spiral packet $\mathrm{P}_{1}$ and seem to be just the tail waves of $P_{1}$. Notice the presence of these shorter-scale waves in the isosurfaces of $w_{i}$ in Fig. 16a. The wave fronts of $w_{i}$ in $\mathrm{P}_{1}$ extend vertically from the surface $z=0$ (Figs. 15b-d) along the curved PV isosurfaces of the vortex, forming a three-dimensional helical structure (Fig. 16a).

A second IGW packet $\left(\mathrm{P}_{2}\right)$, with amplitudes larger than those of $\mathrm{P}_{1}$, originates between $t=6.5$ and $t=7$ $T_{\text {ip }}$ (Figs. 14e and 15e). While packet $\mathrm{P}_{2}$ intensifies in the interior vortex, $\mathrm{P}_{1}$ continues spiraling and dispersing (Figs. $14 \mathrm{~d}-\mathrm{h}$ and $15 \mathrm{~d}-\mathrm{h}$ ). The three-dimensional structure of $\mathrm{P}_{2}$ has several characteristics that distinguish it from $\mathrm{P}_{1}$ (Fig. 16b). In shallow layers the wave fronts of $\mathrm{P}_{2}$ are closed annuli located in horizontal planes, while at deeper layers, they have the threedimensional helical pattern. This circular structure is also observed in Figs. 14g,h. The vertical propagation of $\mathrm{P}_{2}$ is shown in Fig. 17 where, given the large contour interval, the signature of $\mathrm{P}_{1}$ does not appear. The phases of $\mathrm{P}_{2}$ move upward, with a vertical phase speed $\sigma_{Z} \sim 0.09 \pi /\left(5 T_{\mathrm{bp}}\right)=5.9 \times 10^{-2} T_{\mathrm{bp}}^{-1}$, and with local frequency $\omega_{l} \sim 0.5 T_{\mathrm{bp}}^{-1}=0.1 T_{\mathrm{bp}}^{-1}\left(\omega_{l} \sim 0.01\right.$ in flows $\left.A_{i i}-I_{i i}\right)$. These values of $\omega_{l}$ are consistent with the spectra $\left\langle\hat{w}^{2}\right\rangle_{x y}$ discussed in the previous section (Figs. 1012). Since $P_{2}$ originates in the vortex interior (see Figs. 17a and 14e-f), the shallower IGWs of $\mathrm{P}_{2}$ are affected by the vortex circular mean flow (Fig. 17c), resulting in the annular wave fronts. At deeper layers, the vortex mean flow weakens, and the spiral pattern of wave 


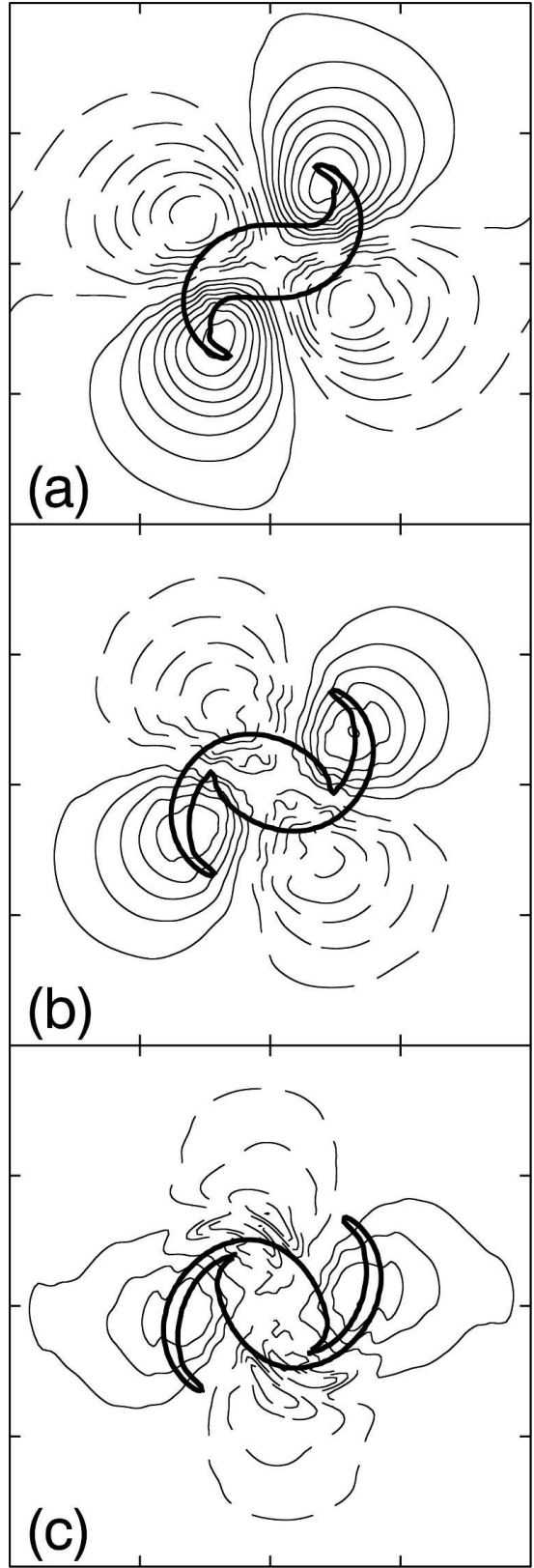

FIG. 13. Horizontal distributions of $w_{b}$ at (a) $t=6 T_{\text {ip }}(w \in$ $\left.[-7.62,8.59] \times 10^{-5}\right)$, (b) $t=7 T_{\text {ip }}\left(w \in[-5.46,6.13] \times 10^{-5}\right)$, and (c) $t=8 T_{\text {ip }}\left(w \in[-5.00,4.00] \times 10^{-5}\right)$ at $z \simeq-1.42$ for the reference case $F_{i}\left(\Delta w_{b}=10^{-5}\right)$.

fronts develops (Fig. 16b). These differences between wave packets $P_{1}$ and $P_{2}$ suggest that they have different origin and presumably a different mechanism of IGW generation.

The wave characteristics can be easily inferred from the Hovmöller diagram of $w_{i}$ for the case $F_{i}$ (Fig. 18). Alternating extrema of $w_{i}(x, t)$ correspond to the crests and troughs of the western IGW packet $\mathrm{P}_{1}$. The averaged local period and horizontal wavelength are
$\Delta T \simeq 7 T_{\mathrm{bp}}$ per cycle and $\Delta L_{x} \simeq 0.85 c$, respectively. This corresponds to an averaged local frequency $\omega=$ $2 \pi / \Delta T=0.14 \times 2 \pi T_{\mathrm{bp}}^{-1}$, which matches the theoretical value of the local frequency of pure plane waves $\omega_{l}=$ $\sqrt{2} f=\sqrt{2} N / c=\sqrt{2} \times 2 \pi / 10$ in the case $m / k=N / f$. The averaged horizontal phase speed $\sigma_{x}=\omega / k=$ $(2 \pi / \Delta T) /\left(2 \pi / \Delta L_{x}\right)=\Delta L_{x} / \Delta T \simeq 0.12 c T_{\mathrm{bp}}^{-1}$.

The time evolution of $\mathbf{u}_{i h}$ on a shallow layer $(z \simeq$ $-0.15)$ is shown in Figs. 19a-c. The spatial average of $\left|\mathbf{u}_{i h}\right|$ in the interior vortex is three orders of magnitude smaller than the domain average of $\left|\mathbf{u}_{h}\right|$, that is, $O\left(\left\langle\left|\mathbf{u}_{h}\right|\right\rangle\right) / O\left(\left\langle\mathbf{u}_{i h} \mid\right\rangle\right)=1 / 10^{-3}=10^{3}$, and therefore the IGWs are not directly perceivable in the $\mathbf{u}_{i}$ distributions (not shown). The magnitude of $\mathbf{u}_{i h}$ increases with time, reaching $\left|\mathbf{u}_{i h}\right|=1.35 \times 10^{-2}$ at $t \simeq 8.5 T_{\mathrm{ip}}$. The $\mathbf{u}_{i h}$ vectors display a periodic pattern of horizontal convergence and divergence (Figs. 19a-c), which is related to the downwelling and upwelling in the vortex (Figs. 19d-f).

The important point is that these IGWs trapped in the vortex interior background flow can eventually cooperate to produce a mean vertical flow. For example, at $t=7 T_{\mathrm{ip}}$, the vectors $\mathbf{u}_{i h}$ have rotated in such a coherent way that produce a net divergence. As a consequence, the water parcels move upward, stretching the isopycnals in the vortex core, and contributing to $\mathcal{F}_{\text {max }}$ (Fig. 2a). However, $\mathcal{F}_{\max }$ is mainly due to the strong vertical shears in the total flow induced by the vortex merging. The origin of $\mathrm{P}_{2}$ seems to be related to the upwelling produced by $w_{i}$ in the shallower layers at $t=7 T_{\text {ip }}$. Flows $E_{i}$ and $D_{i}$ (where $\mathrm{P}_{2}$ is radiated later that in flow $F_{i}$ ) corroborate this positive correlation between the second SAE event and $\mathcal{F}_{\max }$ because the $\mathcal{F}_{\max }$ maximum occurs also later than in case $F_{i}$ (Fig. 2a).

Maximum interaction between the trapped IGWs is produced at $t \simeq 8.4 T_{\mathrm{ip}}$ when the two symmetric packets of $\mathrm{P}_{1}$ interact to generate two strong cells of negative $w_{i}$ (Fig. 20c). This strong vertical motion now releases the upwelled isopycnals so that $\mathcal{F}_{\text {max }}$ decreases (Fig. 2a). Thus, the second and more energetic spontaneous emission seems to be the result of an interaction between preexisting IGWs and the vortex flow. This is a large interaction since the order of magnitude of $\left|w_{i}\right|$ (Fig. 20c) is similar to that of $|w|$ and $\left|w_{b}\right|$, so that $w_{i}$ largely modifies the quadrupolar pattern of $w$ (Figs. 20a,b).

Figure 21 shows the time evolution of $\left\langle\left|w_{i}\right|\right\rangle_{x y}$. In the shallow layers the alternating relative maxima and minima of $\left\langle\left|w_{i}\right|\right\rangle_{x y}$ are related to the SAE events. This oscillating behavior seems to be produced initially by the first SAE event $\left(\mathrm{P}_{1}\right)$, and later by the cooperative interactions between the shallow IGWs and the mean 


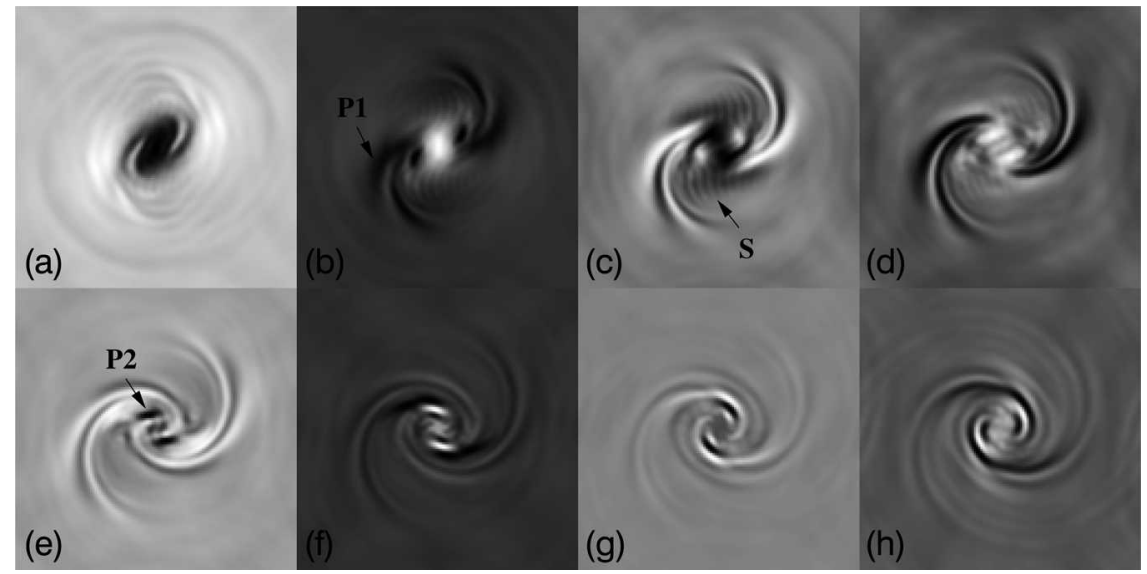

FIG. 14. Horizontal distributions of $w_{i}(\mathrm{a})-(\mathrm{h})$ from $t=5$ to 8.5 every $0.5 T_{\text {ip }}$ on the plane $i_{Z}=51(z \simeq-0.69)$ for the reference case $F_{i}$. The images have $128^{2}$ pixels and extreme values $w_{i} \in\{$ (a) $[-2.37,0.71]$, (b) $[-2.51,5.62]$, (c) $[-3.38,2.65]$, (d) $[-3.16,3.78]$, (e) $[-7.24,3.40]$, (f) $[-7.18,16.0],(\mathrm{g})[-19.9,13.9],(\mathrm{h})[-12.3,14.6]\} \times 10^{-5}$.

flow $\left(\mathrm{P}_{2}\right)$, which, since they depend on the phase rotation of $\mathbf{u}_{i h}$, are produced periodically. First, the rotating background flow generates IGWs (basically inertial waves in background flow) whose phases are initially and spatially modulated by the rotating flow. As time evolves these spatially coherent IGWs can produce large-scale horizontal divergence, rising up the upper isopycnals in the vortex core and generating the wave packet $\mathrm{P}_{2}$. This motion is eventually limited by the radial pressure gradient force and by the phase rotation of the IGW velocity (which now, a $T_{\mathrm{ip}} / 4$ later, point tangent to the circular background flow). A $T_{\mathrm{ip}} / 4$ later the IGW velocity is convergent forcing the downward motion of isopycnals, which stop descending a $T_{\mathrm{ip}} / 4$ later when $\mathbf{u}_{i h}$ is again tangent to the circular background flow, so that the fluid is ready for a new IGW emission. This process repeats about every $T_{\mathrm{ip}}$.

Previous to the second type of SAE event, $\left\langle\left|w_{i}\right|\right\rangle_{x y}$ increases in the shallow layers because of the wavemean flow interaction, but immediately later it decreases since the IGW packet propagates away from the vortex. This downward propagation of the IGW packets is clear in Fig. 21, where the contours of $\left\langle\left|w_{i}\right|\right\rangle_{x y}(z, t)$ have negative vertical slopes, with the downward advection of $P_{2}$ larger than that of $P_{1}$.

The total energy $\left(E_{T}\right)$ can be decomposed as follows:

$$
E_{T}=\boldsymbol{u}^{2}+N^{2} \mathcal{D}^{2}=E_{T b}+E_{T i}+E_{T \text { int }},
$$

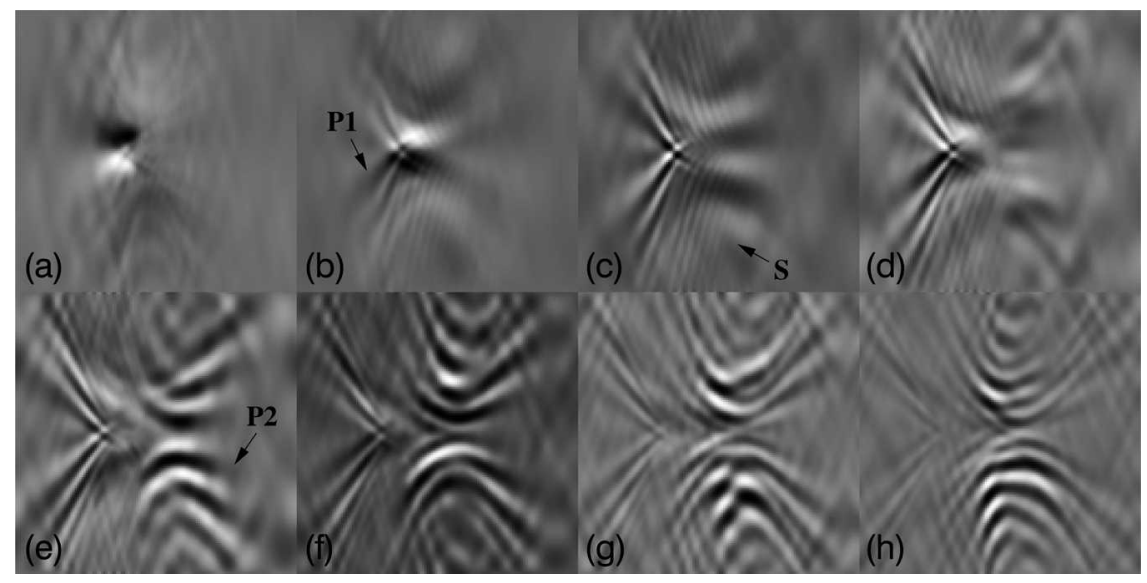

FIG. 15. Vertical distributions of $w_{i}$ (a)-(h) from $t=5$ to 8.5 every $0.5 T_{\text {ip }}$ at $y \simeq-0.93 c$ for the reference case $F_{i}$. The images have $128^{2}$ pixels and extreme values $w_{i} \in\{(\mathrm{a})[-1.97,1.75]$, (b) $[-4.60,4.63]$, (c) $[-3.03,3.33]$, (d) $[-3.19,2.83]$, (e) $[-2.80,2.65]$, (f) $[-2.85,3.96]$, (g) $[-5.20,4.55]$, (h) $[-9.56,8.84]\} \times 10^{-5}$. 

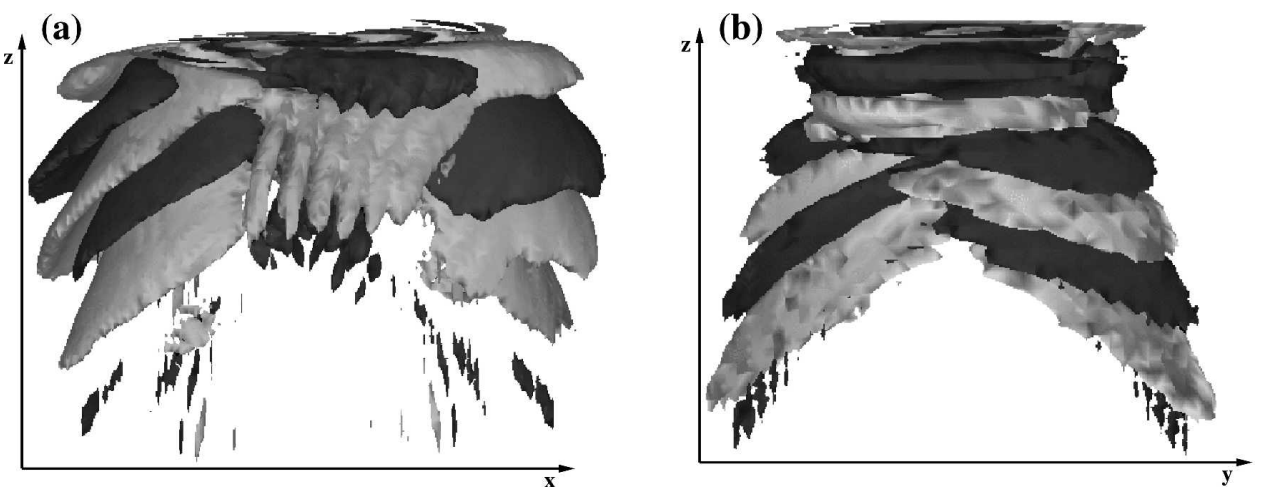

FIG. 16. Isosurfaces of (a) $w_{i}= \pm 10^{-5}$ at $t=6 T_{\text {ip }}$ (south view, $x \in[-0.8,0.9] c ; z \in[-2.75,0]$ ), and (b) $w_{i}= \pm 10^{-4}$ at $t=8 T_{\text {ip }}$ (west view; $y \in[-1.9,2] c ; z \in[-1.7,0]$ ). Dark gray means $w_{i}>0$.

where $\boldsymbol{u}^{2}$ is twice the kinetic energy, $N^{2} \mathcal{D}^{2}$ is twice the potential energy, $E_{T b}=\boldsymbol{u}_{b}^{2}+N^{2} \mathcal{D}_{b}^{2}$ is the total energy of the balanced flow, $E_{T i}=\boldsymbol{u}_{i}^{2}+N^{2} \mathcal{D}_{i}^{2}$ is the total energy of the unbalanced flow, and $E_{T \text { int }}=2 N^{2} \mathcal{D}_{b} \mathcal{D}_{i}+$ $2 \boldsymbol{u}_{b}^{2} \boldsymbol{u}_{i}^{2}$ is the interaction term between the balanced and unbalanced flows. The total energy is not the sum of the balanced and unbalanced components of the flow since the kinetic and potential energy are quadratic functions of $\boldsymbol{u}$ and $\mathcal{D}$, respectively. The time evolution of the total energy is shown in Fig. 22. The magnitude of $E_{T}, E_{T b}$, and $E_{T \text { int }}$ decreases with time; meanwhile $E_{T i}$ increases, which indicates the energy transfer from the total flow to the IGWs (Fig. 22).

We consider two different ways to estimate the energy lost from the total flow. The first one assumes that during the first three buoyancy periods (from $t=5.1$ to $t=5.4 T_{\mathrm{ip}}$ ) the total energy is very well conserved so that there is no energy lost by numerical diffusion. In this case the ratio (in percentage) between the energy transfer per unit time $\left(E_{T \text { int }} / 3 T_{\mathrm{bp}}\right)$ and the total energy $E_{T}$ is $\left(100 \times E_{T \text { int }}\right) /\left(E_{T} \times 3 T_{\mathrm{bp}}\right) \simeq 0.26 \% T_{\mathrm{ip}}^{-1}$, or about
$2.6 \%$ per rotation period. The second estimate assumes that the decrease of the total energy after $T=5.4 T_{\mathrm{ip}}$ is transferred to the wave motion, then to the grid size scales, and finally lost by numerical diffusion. In this case the energy transfer is $\left(100 \times \Delta E_{T}\right) /\left(\Delta t E_{T}\right) \simeq 0.7 \%$, or $7 \%$ per rotation period. The order of magnitude of these values are in agreement with the $4 \%$ estimated by Afanasyev (2003) after a dipole collision in laboratory experiments, and the $1 \%$ obtained by Williams et al. (2007, manuscript submitted to J. Atmos. Sci.). Since the ocean's dynamics is mainly driven by a large quantity of mesoscale coherent vortical flows, the spontaneous emission of IGWs can represent a robust phenomena radiating energy to the deep ocean, and if so, it should be taken into account in the parameterizations of the turbulent closure models.

\section{Indicators of SAE events}

This section addresses the relations between the two IGW packets, $\mathrm{P}_{1}$ and $\mathrm{P}_{2}$, described above and possible

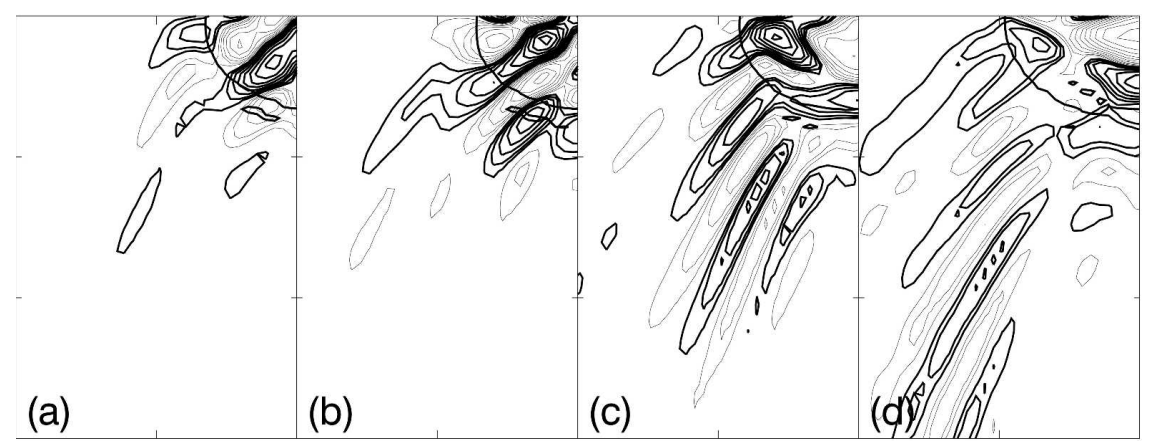

FIG. 17. Closer view of the vertical distributions of $w_{i}$ at (a) $t=7 T_{\mathrm{ip}}$, (b) $t=7.5 T_{\mathrm{ip}}$, (c) $t=8 T_{\mathrm{ip}}$, and (d) $t=8.5 T_{\mathrm{ip}}$ at $x=0$ (thick contours mean $w_{i}>0$, contour levels equal to $\left.\{\ldots,-4,-3,3,4, \ldots\}^{2.5} \times 10^{-6}\right)$. Domain is $x \in[-\pi / 2,0] c$ and $z \in[-3 \pi / 4,0]$. The PV contour $\varpi=-0.01$ (thick solid line) is included. 


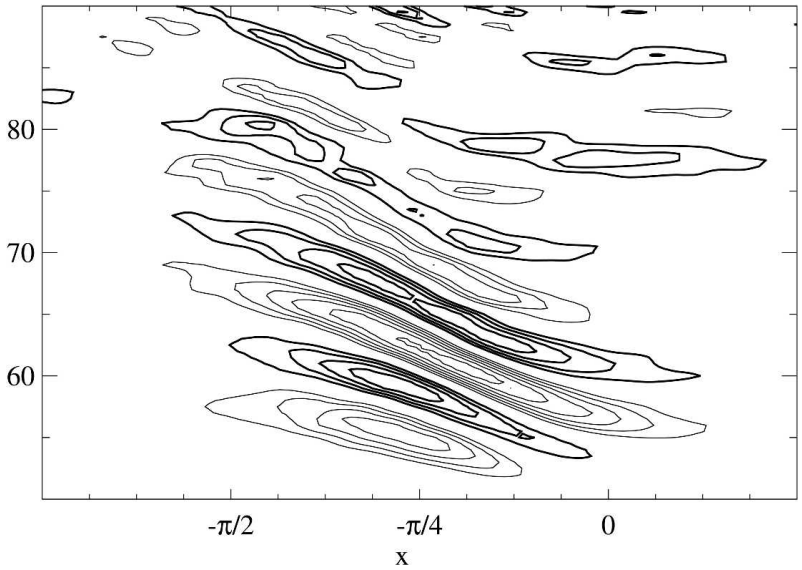

FIG. 18. Hovmöller diagram of $w_{i}(x, t)$ at grid point $\left(i_{y}, i_{z}\right)=\{32$, 60 for the case $F_{i}$ (thick contours mean $w_{i}>0$, minimum contour $4 \times 10^{-6}$ with $\left.\Delta=2 \times 10^{-6}\right)$. Vertical axis is time in buoyancy periods.

theoretical indicators of IGW generation. Three indicators are considered, namely,

(i) the horizontal advection of PV $\left(\mathbf{u}_{h} \cdot \nabla_{h} \varpi\right)$;

(ii) the residual of the nonlinear balance equation $\Delta_{\text {nbe }}$ (or the approximated divergence tendency); and

(iii) the Lighthill-Ford radiation term.

\section{a. The advection of $P V$}

It is plausible that, since the anticyclone is defined as a given amount of PV anomaly on a constant PV background fluid (which involves density gradients), the local changes due to the displacement of the outer isosurfaces of $\varpi$ may perturb the background flow. If this perturbation is large enough it may generate waves, in a way similar to the waves produced by a dense body moving on still water. We suggest that this mechanism is related to the origin of the first (weaker) IGW packet $\mathrm{P}_{1}$.

To address this possibility the horizontal advection of $\mathrm{PV}$ of the total flow is plotted in the interior vortex, together with $w_{i}$ (Figs. 23a-c). The $\mathbf{u}_{h} \cdot \nabla_{h} \varpi$ has a relative maximum and an absolute minimum near the edge of the vortex. These extrema seem to be related to the large horizontal PV gradients (Fig. 1e) since there is a good spatial correspondence between the location of these extrema and the location of the IGWs. The curvature of the wave fronts matches the curvature of the PV advection maxima; both structures rotate clockwise with time and the wavelength of the IGWs increases with the distance from these PV advection maxima (Figs. 23a,b). These characteristics are consistent along the fluid column (Figs. 23d-f). Thus, the IGW packet $P_{1}$ originates at the surface layers where there is large PV

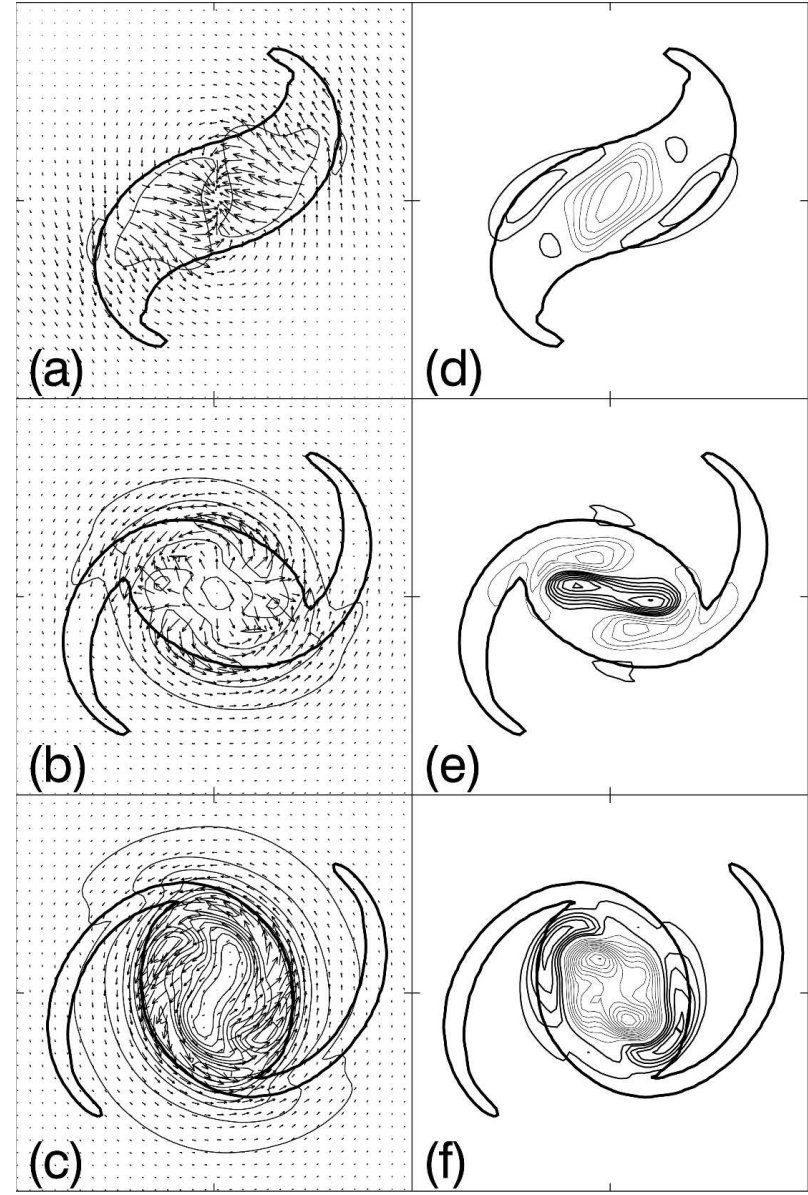

FIG. 19. Closer view of the horizontal distributions of (a)-(c) $\mathbf{u}_{i h}$ (contours of $\left|\mathbf{u}_{i h}\right|, \Delta\left|\mathbf{u}_{i h}\right|=1.5 \times 10^{-3}$ ) and (d)-(f) $w_{i}$ (thick lines mean $w_{i}>0, \Delta w_{i}=7.5 \times 10^{-5}$ ), at $z \simeq-0.15$ and (a), (d) $t=5.5$ $T_{\text {ip }}$, (b), (e) $t=7 T_{\text {ip }}$, and (c), (f) $t=8.5 T_{\text {ip }}$. Domain is $x, y \in$ $[-\pi / 2, \pi / 2] c$. The PV contour $\varpi=-0.01$ (thick solid line) is included.

advection. A second relative minimum of $\left|\mathbf{u}_{h} \cdot \nabla_{h} \varpi\right|$ located on the rear of the vortex (Figs. 23a-c) may be related to the small perturbations in $w_{i}$ of the IGW packet $\mathrm{S}$.

Another example of SAE related to the PV advection, occurring at a much later time, is shown in Fig. 24. IGWs are generated on both apexes of the ellipsoid where the local change of PV is larger. This is in agreement with an enhancement of the spatial average of $\mathbf{u}_{h} \cdot \nabla_{h} \varpi$ happening between $t=15$ and $17 T_{\text {ip }}$ (not shown). This IGW packet was not further analyzed because of the interaction with the mirror waves generated previously $\left(\mathrm{P}_{1}\right.$ and $\left.\mathrm{P}_{2}\right)$.

\section{b. Approximated divergence tendency equation}

Applying the horizontal divergence operator to the horizontal momentum equation 


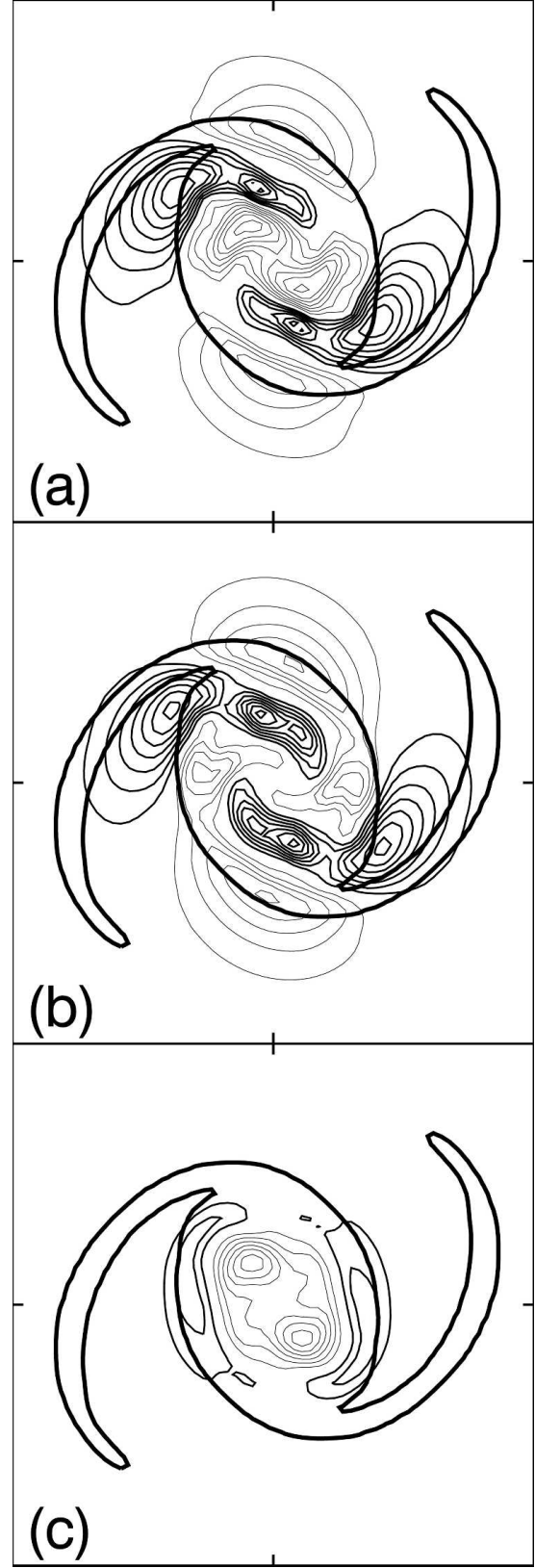

FIG. 20. Horizontal distributions of (a) $w(w \in[-1.13,1.19] \times$ $\left.10^{-4}\right)$, (b) $w_{b}\left(w_{b} \in[-0.77,1.40] \times 10^{-3}\right)$, and (c) $w_{i}\left(w_{i} \in[-1.07\right.$, $\left.0.37] \times 10^{-3}\right)$ at $z \simeq-0.15$, and $t=8.4 T_{\text {ip }}$ (thick contours mean positive values, contour interval $\Delta=1.5 \times 10^{-4}$, zero contour omitted). The PV contour $\varpi=-0.01$ (thick solid line) is included.

$$
\frac{d \mathbf{u}_{h}}{d t}+f \mathbf{k} \times \mathbf{u}_{h}^{\prime}=0,
$$

where $\mathbf{u}_{h}^{\prime}$ is the ageostrophic velocity, the divergence tendency equation may be written as

$$
\frac{d\left(-w_{z}\right)}{d t}=2 g_{X Y}\{u, v\}-\nabla w \cdot \mathbf{u}_{z}+f \zeta^{\prime},
$$

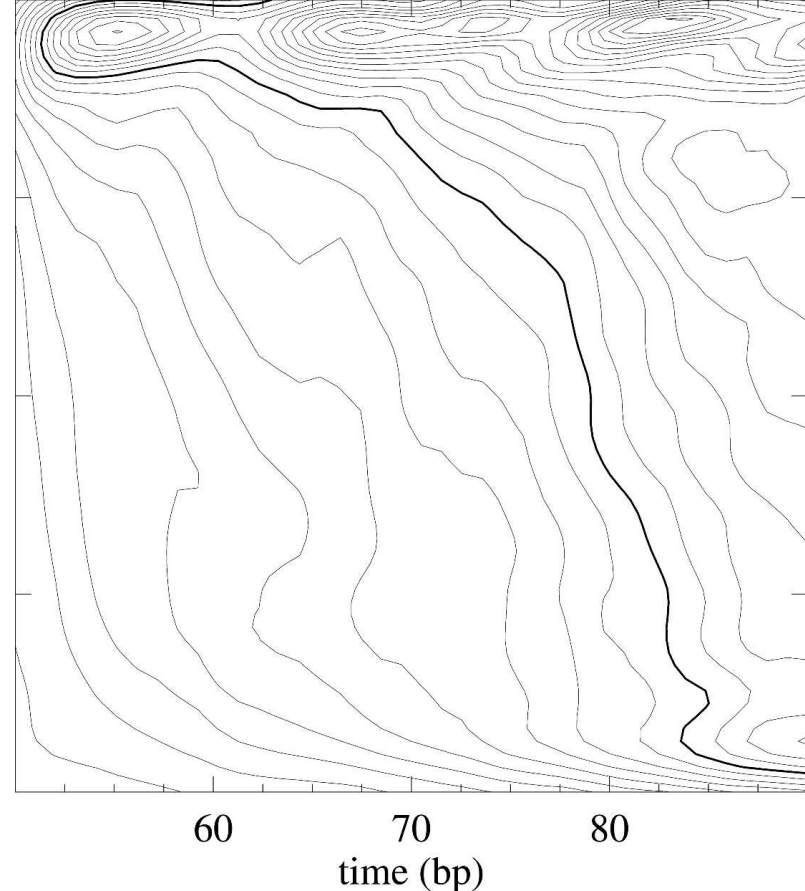

FIG. 21. Contours of the horizontal average $\left\langle\left|w_{i}\right|\right\rangle_{x y}(z, t)$ as a function of depth and time (thick line is the contour $\left\langle\left|w_{i}\right|\right\rangle_{x y}$ $\left.(z, t)=5 \times 10^{-6}, \Delta=5 \times 10^{-7}\right)$. Vertical axis is $z \in[-\pi / 2,0]$.

where $\mathcal{I}_{X Y}\{u, v\} \equiv u_{x} v_{y}-v_{x} u_{y}$ is the horizontal Jacobian, subscripts $x, y$, and $z$ mean the partial derivative, and $\zeta^{\prime} \equiv \mathbf{k} \cdot \boldsymbol{\nabla} \times \mathbf{u}^{\prime}$ is the ageostrophic vertical vorticity.

Term $\boldsymbol{\nabla} w \cdot \mathbf{u}_{z}$ on the right-hand side (rhs) of (6) was found to be two orders of magnitude smaller than the other two terms and is neglected. Hence, a good approximation to (6) is

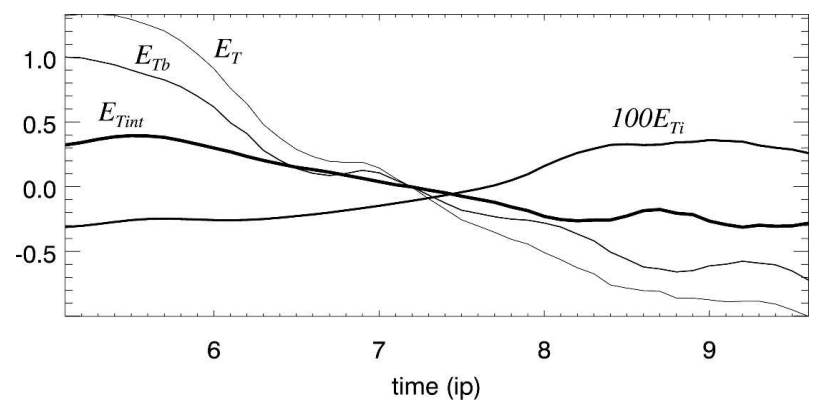

FIG. 22. Time evolution of the total energy $E_{T}$, total energy of the balanced flow $E_{T b}$, scaled total energy of the unbalanced flow $100 E_{T i}$, and the interaction term between balanced and unbalanced flow $E_{\text {Tinn }}$. The vertical axis is in units of $10^{-4}$. The slope for $E_{T}$, obtained from a fitted linear model by minimizing the chi-square error statistics, is $\Delta E_{T} / \Delta t \simeq-5.5 \times 10^{-5} T_{\mathrm{ip}}^{-1}$. For comparison purposes the plots represent $E_{\chi} \equiv\left\langle E_{\chi}\right\rangle-\overline{\left\langle E_{\chi}\right\rangle}$, where $\overline{\left\langle E_{\chi}\right\rangle}$ is the time average of the spatial average $\left\langle E_{\chi}\right\rangle$. The time averages and the standard deviations are $\overline{\left\langle E_{T}\right\rangle}=$ $(75 \pm 0.8) \times 10^{-4},\left\langle\overline{\left.E_{T b}\right\rangle}=(75 \pm 0.5) \times 10^{-4},\left\langle\overline{\left.E_{T \text { int }}\right\rangle}=\right.\right.$ $(-0.4 \pm 0.2) \times 10^{-4}$, and $\overline{\left\langle E_{T i}\right\rangle}=(3 \pm 2) \times 10^{-7}$. 

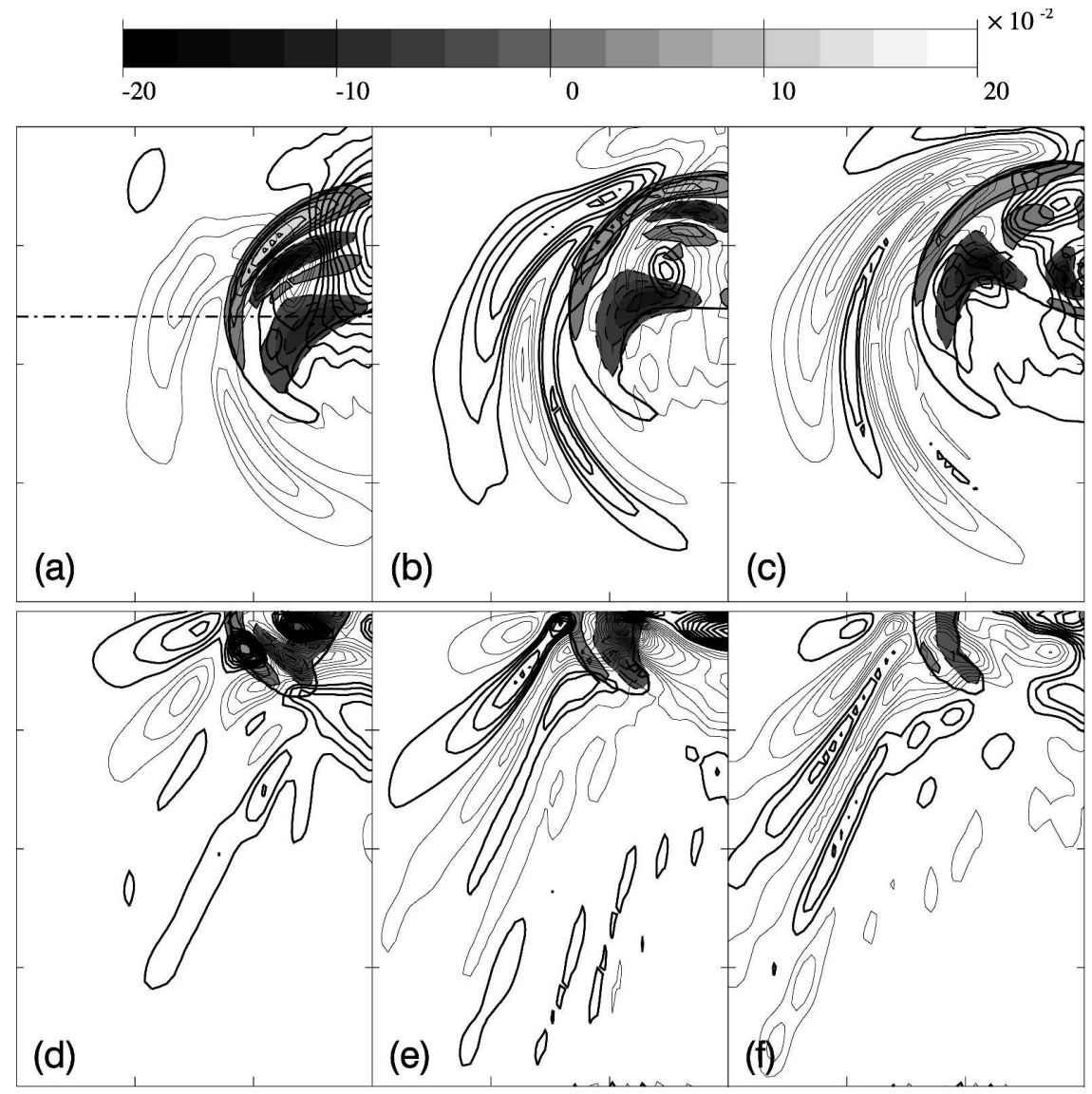

Fig. 23. Closer view of the (a)-(c) horizontal $(z \simeq-0.69)$ and (d)-(f) vertical $(y \simeq-0.44 c)$ distributions of $w_{i}$ at (a), (d) $t=5.5$, (b), (e) $t=6$, and (c), (f) $t=6.5 T_{\text {ip }}$ (thick contours mean $w_{i}>0, \Delta=0.5 \times 10^{-5}$ ). The advection of PV at $z=0$ is included (solid/dashed contours mean positive/negative values of $\mathbf{u}_{h} \cdot \nabla_{h} \varpi, \Delta=2.5 \times 10^{-2}$ ). The PV contour $\varpi=-0.01$ (thick solid line) is included. Domain extent is $(x, y) \in[-3 \pi / 4,0] c$, and $z \in[-\pi /, 0]$.

$$
\frac{d\left(-w_{z}\right)}{d t}=\Delta_{\text {nbe }}
$$

where

$$
\Delta_{\text {nbe }} \equiv 2 g_{X Y}\{u, v\}+f \zeta^{\prime}
$$

is referred to as the residual of the nonlinear balance equation. This quantity has been recently used to diagnose flow imbalance in order to quantify the generation of gravity waves in atmospheric baroclinic jet fronts (Zhang et al. 2001; Zhang 2004). Since $\Delta_{\text {nbe }}$ is an approximated measure of the material rate of change of the horizontal divergence (a quantity smaller that $\zeta^{\prime}$ in mesoscale balanced flows), there is a large cancellation between terms $2 \mathcal{I}_{X Y}\{u, v\}$ and $f \zeta^{\prime}$. Hence, not only is the rate of change of $w_{z}$ of the balanced flow present in the $\Delta_{\text {nbe }}$ distribution, but the rate of change of $w_{i z}$ of the IGWs is also present.

The field $\Delta_{\text {nbe }}$ was analyzed at times when the packets $\mathrm{P}_{1}$ and $\mathrm{P}_{2}$ appear, although we focus next in the generation of $\mathrm{P}_{2}$ since it is the strongest $\mathrm{SAE}$ event. Figure 25 shows the time evolution of $\Delta_{\text {nbe }}$ at two different layers. At deep layers, $\Delta_{\text {nbe }}$ seems to identify unambiguously the timing and location of the IGWs, which is in agreement with the distributions of $w_{i}$ (Figs. $14 \mathrm{e}-\mathrm{h}$ ). Hence, the IGWs radiated spontaneously could be detected experimentally if very accurate horizontal total velocity $\mathbf{u}_{h}$ and density $\rho$ are known. At surface layers, however, the magnitude of the $\Delta_{\text {nbe }}$ indicator may be attributed to the convergence (divergence) pattern observed in Figs. 19a-c. At $z=0, \Delta_{\text {nbe }}$ increases in the vortex interior between $t=6.5$ and $7 T_{\mathrm{ip}}$, the time at which appears the first evidence of $\mathrm{P}_{2}$ at $z=-0.69$ (Figs. 14e and 15e). Extrema of $\Delta_{\text {nbe }}$ occur at $t \simeq 7.5 T_{\text {ip }}$, with diminishing magnitude at later times $t=8$ and 8.5 $T_{\mathrm{ip}}$. Location and timing of these maxima suggests that the emission of $\mathrm{P}_{2}$ is related to the coherent phase motion (as induced by the balanced anticyclonic largescale flow) of the preexisting IGWs at shallower layers. 


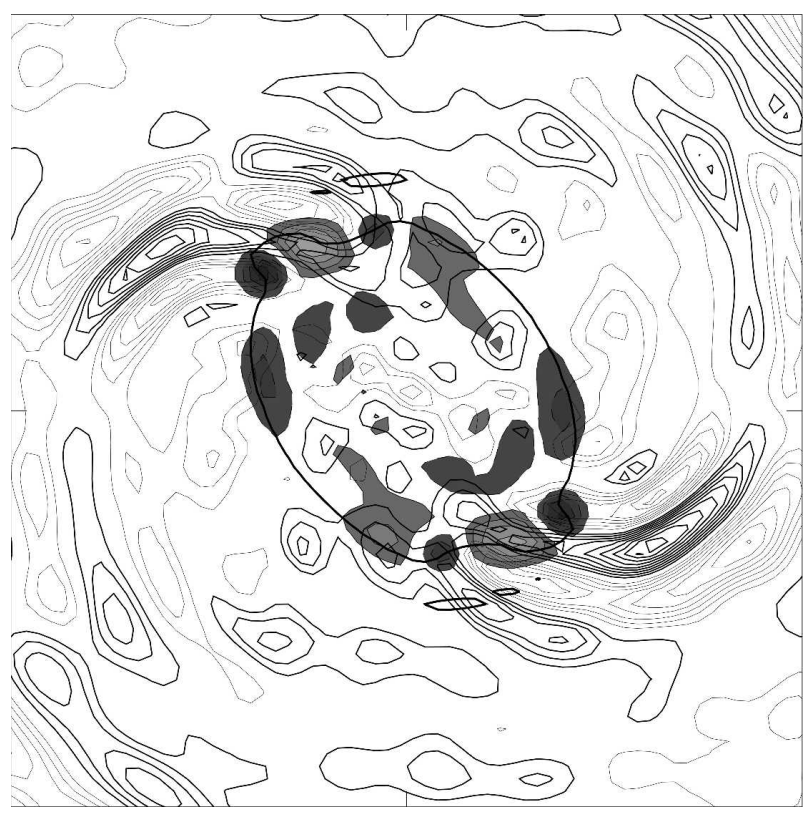

FIG. 24. Horizontal distributions at $t=17 T_{\mathrm{ip}}$ of $-w_{z}(z=$ $\left.-1.42,-w_{z} \in[-4.87,5.81] \times 10^{-4}, \Delta=6 \times 10^{-5}\right)$ and the advection of PV (shaded, $z=0, \mathbf{u}_{h} \cdot \nabla_{h} \varpi \in[-0.11,0.12], \Delta=$ $\left.2.5 \times 10^{-2}\right)$.

If the mechanism mentioned above stimulates the emission of $\mathrm{P}_{2}$, the maximum amplitude reached at $t \simeq$ $8-8.5 T_{\mathrm{ip}}$ at $z=-69$ (Figs. 14g,h) may be correlated to the large values of $\Delta_{\text {nbe }}$ at $t \simeq 7-7.5 T_{\text {ip }}$ at $z=0$ (Figs. $25 \mathrm{a}, \mathrm{b})$. Using the estimated vertical phase speed for this wave packet (section 6) it takes $t \simeq 0.69 / 0.06 \simeq 11 T_{\mathrm{bp}}$ for $\mathrm{P}_{2}$ to propagate downward the distance $\Delta z=-0.69$. However, the phase speed in section 6 was computed for a freely propagating wave packet with no background flow (Figs. 17c,d), and this strongest propagating wave is generated in the vortex interior. Thus, there is an additional time interval of about $\Delta t \simeq 5-6 T_{\mathrm{bp}}$ for $\mathrm{P}_{2}$ to propagate through the vortex vertical extent (Fig. 21).

\section{c. The extended Lighthill-Ford source term}

The Lighthill-Ford source term has been proposed as an indicator of the spontaneous generation of IGWs in shallow waters systems (Ford 1994; Williams et al. 2005). This theoretical development based on the analogy with the theory of sound generated aerodynamically (Lighthill 1952) and the shallow-water equations can be generalized to the three-dimensional Boussinesq equations as follows. Since

$$
\boldsymbol{\nabla}_{h} \cdot \mathcal{A}_{h}=-\frac{1}{f} \zeta_{z}^{\prime}, \quad \text { and } \quad \mathbf{k} \cdot \boldsymbol{\nabla} \times \mathcal{A}_{h}=-\frac{1}{f} \nabla^{2} w
$$

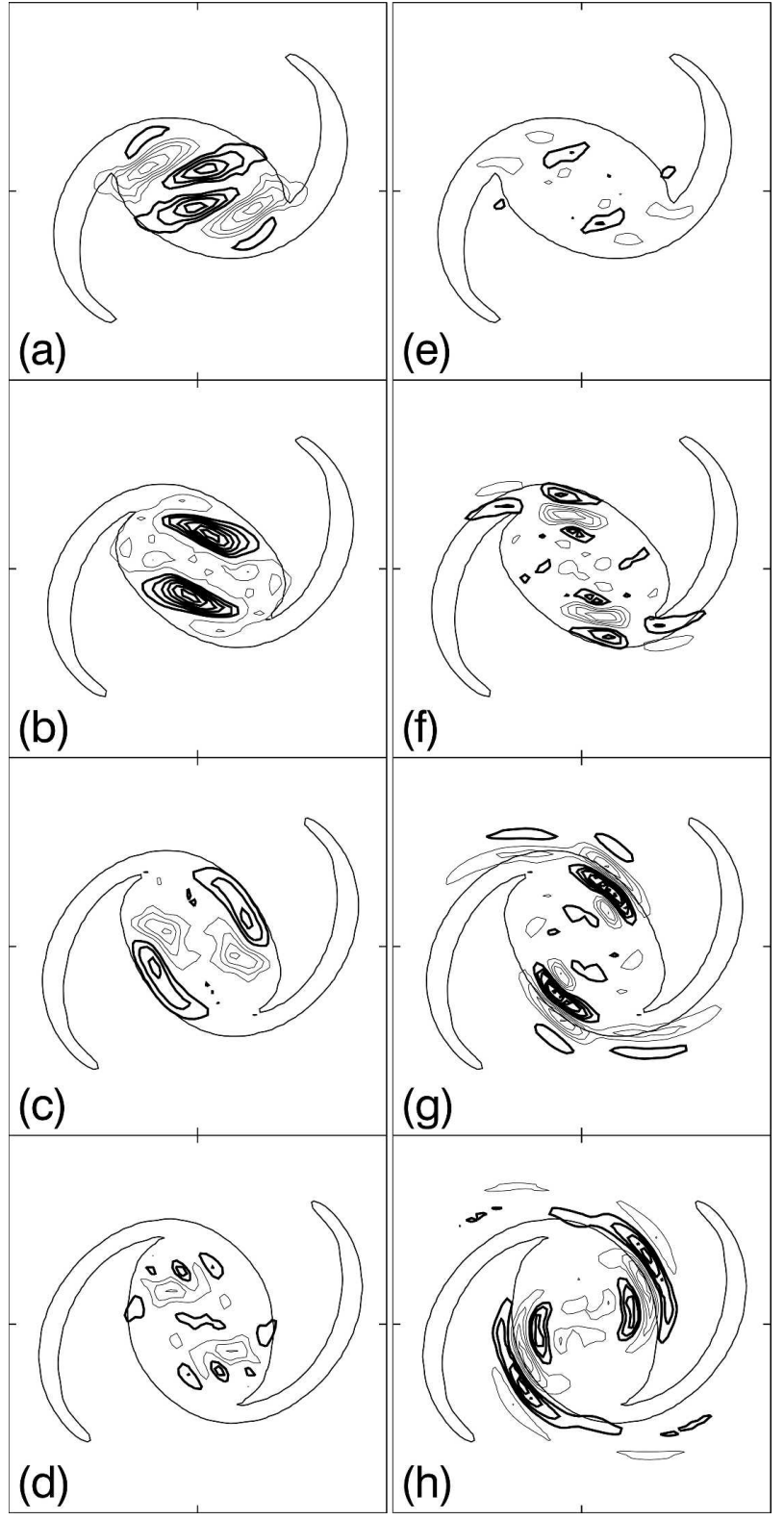

FIG. 25. Horizontal distributions of $\Delta_{\text {nbe }}$ at (a)-(d) $z=0(\Delta=$ $\left.2.5 \times 10^{-3}\right)$ and $(\mathrm{e})-(\mathrm{g}) z=-0.69\left(\Delta=0.5 \times 10^{-3}\right)$ at $(\mathrm{a}),(\mathrm{e})$ $t=7 T_{\mathrm{ip}}$, (b), (f) $t=7.5 T_{\mathrm{ip}}$, (c), (g) $t=8 T_{\mathrm{ip}}$, and (d), (h) $t=8.5$ $T_{\text {ip }}$. Domain is $x, y \in[-\pi / 2, \pi / 2] c$. The PV contour $\varpi=-0.01$ (thick solid line) is included.

the divergence of (3) results in the generalized omega equation

$$
\begin{aligned}
\frac{1}{f} \frac{d \zeta_{z}^{\prime}}{d t}= & -\nabla_{h} \cdot\left[2 \mathbf{Q}_{h}+c^{2}\left(\mathcal{D}_{z}-1\right) \boldsymbol{\nabla}_{h} w\right]+\left(\frac{\zeta}{f}+1\right) w_{z z} \\
& +\frac{1}{f} \boldsymbol{\zeta}_{h z}^{\prime} \cdot \nabla_{h} w+\frac{1}{f} \boldsymbol{\zeta}_{h}^{\prime} \cdot \nabla_{h}^{2} \mathbf{u}_{h},
\end{aligned}
$$

where $\mathbf{Q}_{h} \equiv c^{2} \boldsymbol{\nabla}_{h} \mathbf{u}_{h} \cdot \boldsymbol{\nabla}_{h} \mathcal{D}$ is the generalized $\mathbf{Q}$ vector, and $\zeta_{h}^{\prime} \equiv \mathbf{k} \times \boldsymbol{u}_{h z}^{\prime}=-v_{z}^{\prime} \mathbf{i}+u_{z}^{\prime} \mathbf{j}$ is the ageostrophic hori- 
zontal pseudovorticity (i.e., the vorticity of the horizontal ageostrophic velocity).

The vertical component of the curl of (3) provides the equation for the rate of change of $\nabla^{2} w$,

$$
\frac{d}{d t}\left(-\nabla^{2} w\right)=f \zeta_{z}^{\prime}+\nabla_{h} \mathbf{u}:\left[\nabla_{h} \nabla w-\left(\nabla \mathbf{u}_{h z}\right)^{\mathrm{T}}\right],
$$

where $\mathbf{A}: \mathbf{B}=\operatorname{tr}\left(\mathbf{A B}^{\mathrm{T}}\right)=\Sigma A_{i j} B_{i j}$ is the scalar "double dot" product of two dyadics $\mathbf{A}=\Sigma A_{i j} \boldsymbol{e}_{i} \boldsymbol{e}_{j}$ and $\mathbf{B}=\Sigma$ $B_{i j} \boldsymbol{e}_{i} \boldsymbol{e}_{j}$, where $\mathbf{e}_{i}$ and $\mathbf{e}_{j}$ are the base vectors. The rate of change of $\zeta_{z}^{\prime}$ in (9) can be eliminated using the rate of change of (10), and grouping the linear terms we obtain

$$
\frac{d^{2}}{d t^{2}} \nabla^{2} w+N^{2} \nabla_{h}^{2} w+f^{2} w_{z z}=\mathcal{N}_{\mathcal{L}},
$$

where the nonlinear term

$$
\begin{aligned}
\mathcal{N}_{L} \equiv & -\boldsymbol{\nabla}_{h} \cdot\left(2 \mathbf{Q}_{h}+\mathcal{D}_{z} \boldsymbol{\nabla}_{h} w\right)-\frac{\zeta}{f} w_{z z}-\frac{\zeta^{\prime}{ }_{h z}}{f} \cdot \nabla_{h} w \\
& +\frac{\zeta^{\prime}{ }_{h}}{f} \cdot \nabla_{h}^{2} \mathbf{u}_{h}-\frac{d}{d t}\left\{\boldsymbol{\nabla}_{h} \mathbf{u}:\left[\nabla_{h} \boldsymbol{\nabla} w-\left(\nabla_{\mathbf{u}_{h z}}\right)^{\mathrm{T}}\right]\right\} .
\end{aligned}
$$

For linear complex plane wave solutions of the form

$$
\hat{\chi}(\mathbf{x}, t) \equiv \hat{\chi}_{0} e^{i(\boldsymbol{\kappa} \cdot \mathbf{x}-\omega t)},
$$

term $\mathcal{N}_{L}$ and the nonlinear terms in $d^{2}\left(\nabla^{2} w\right) / d t^{2}$ in (11) are zero, and the dispersion relation for linear plane waves is directly obtained:

$$
\omega^{2}=\frac{N^{2}\left(k^{2}+l^{2}\right)+f^{2} m^{2}}{k^{2}+l^{2}+m^{2}},
$$

where the wavenumber vector $\boldsymbol{\kappa}=(k, l, m)$. Therefore (11) is a generalization of the rotating shallow water IGW Lighthill generation theory to three-dimensional baroclinic Boussinesq flows.

For linear plane wave solutions the rhs of (11) equals zero, so $\mathcal{N}_{\mathcal{L}}$ can be interpreted as a deviation from linear plane wave solutions. However, the relevant question is the following: Can $\mathcal{N}_{\mathcal{L}}$ in (11) be interpreted as the source term of IGWs? Let us assume that IGWs are not spontaneously generated in the QG regime. Thus, a difficulty with the interpretation above is that in the QG limit the nonlinear QG term $\mathcal{N}_{\mathcal{L}}^{q}$, that is, the QG approximation to $\mathcal{N}_{\mathcal{L}}$, includes the divergence of the geostrophic $\mathbf{Q}$ vector $\mathbf{Q}_{h}^{g} \equiv c^{2} \boldsymbol{\nabla}_{h} \mathbf{u}_{h}^{g} \cdot \boldsymbol{\nabla}_{h} \mathcal{D}$, where $\mathbf{u}_{h}^{g}$ is the geostrophic velocity. This term is balanced by the QG terms $N^{2} \nabla_{h}^{2} w^{q}+f^{2} w_{z z}^{q}$ involving the QG vertical velocity $w^{q}$ in the left-hand side of (11), so that the QG $\omega$ equation holds:

$$
c^{2} \nabla_{h}^{2} w^{q}+w_{z z}^{q}=2 \nabla_{h} \cdot Q_{h}^{g} .
$$

Thus, term $\mathcal{N}_{\mathcal{L}}$ includes QG terms and is therefore different from zero in balanced flows. We note that $\mathcal{N}_{\mathcal{L}}$ is also different from zero in the case of nonplane (spherical, spiral, etc.) IGWs freely propagating far away from the vortical regions where they have been generated.

If, however, infinitely small amplitude IGWs are always generated in nonstationary flows, including the limit $R \rightarrow 0$, the above criticism does not apply. The conjecture of universal IGW generation is becoming gradually accepted, although not fully demonstrated (Saujani and Shepherd 2002), based on studies on the nonexistence of an exact solution for the Lorenz superbalance equation (Vautard and Legras 1986; Lorenz and Krishnamurthy 1987; Warn 1997), and on loworder models of the Boussinesq equations (e.g., Lorenz 1986; Vanneste and Yavneh 2004). Furthermore, if this universal IGW generation does not occur, the same criticism applies also to the indicators based on $\mathbf{u}_{h} \cdot \nabla_{h} \boldsymbol{\varpi}$ and $\Delta_{\text {nbe }}$, which are different from zero in the QG limit. Thus, these indicators would be only approximations, as long as a threshold value for wave generation is missing, to the IGW generation source. The final, unified criterion for the spontaneous generation of IGWs has still to be found. If, however, the universal IGW generation is true the three indicators are partially valid depending on the generation mechanism.

Term $\mathcal{X}_{\mathcal{L}}$ is large and axis-symmetrical inside the vortex, and displays a quadrupolar pattern at the early times (Figs. 26a,b), coincident with the spontaneous generation of $\mathrm{P}_{1}$ (between $t=4$ and $t=5 T_{\mathrm{ip}}$ ). At $t=$ $6 T_{\text {ip }}$ (Fig. 26c) and at later times, the distribution of $\mathcal{N}_{\perp}$ is noisier due to the large number of derivatives involved in $\mathcal{N}_{L}$. We in fact computed $\mathcal{N}_{L}$ as the left-hand side of Eq. (11), where the dominant term is $\partial^{2} w_{z z} / \partial t^{2}$. It is, however, not easy to find an evident correlation between the pattern of $\mathcal{N}_{\mathcal{L}}$ and the generation of the spiral IGW packets.

\section{Concluding remarks}

Several events of spontaneous generation of shortscale inertia-gravity waves emitted during the merging of two anticyclonic baroclinic vortices have been numerically investigated. The results show that this spontaneous generation of IGWs occurs in flows with different values of parameters, namely, the maximum dimensionless PV anomaly of the anticyclones $\left(\left|\varpi_{0}^{-}\right|\right)$, vortex aspect ratio $\left(a_{X} / a_{Z}\right)$, background rotation $(N / f)$, numerical diffusion, and numerical resolution. The larger the relative vorticity (related to a large amount of PV), and the larger the spatial isotropy (ratio $N / f$ closer to 1), the larger the energy of the spontaneous emission events. 


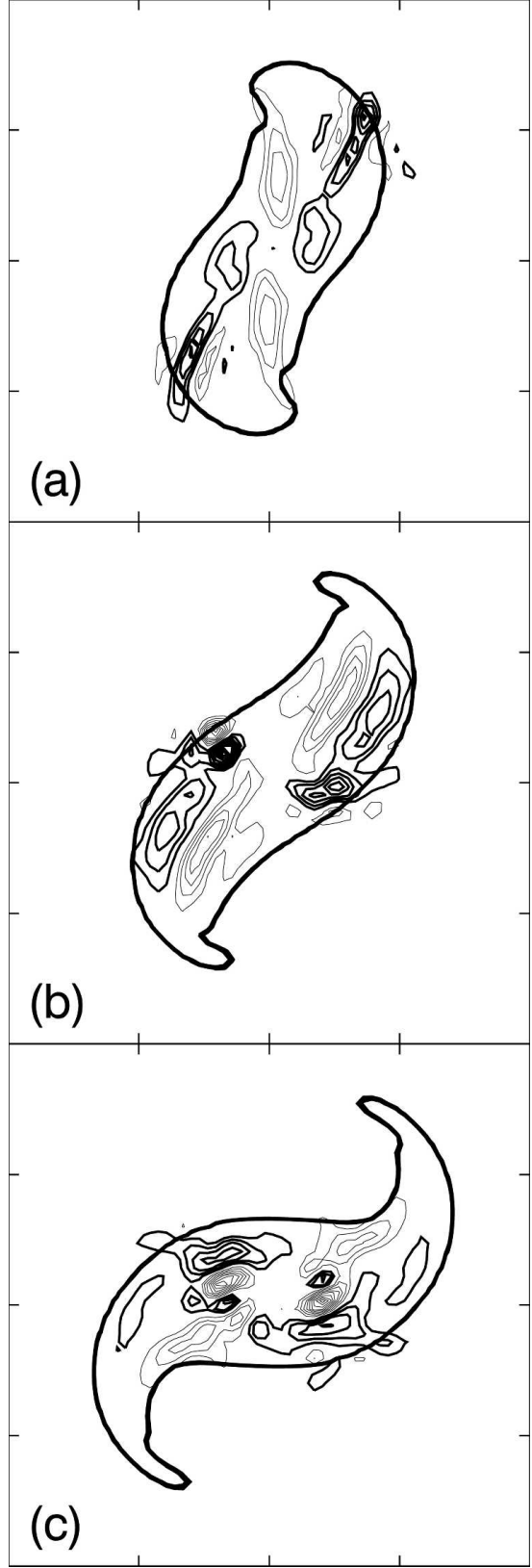

FIG. 26. Horizontal distributions of $\mathcal{N}_{\mathcal{L}}$ at $z=-0.24$ (thick contours mean positive values, contour interval $\Delta=6 \times 10^{-2}$, zero contour omitted), at (a) $t=4 T_{\text {ip }}\left(\mathcal{N}_{L} \in[-0.23,3.22]\right)$, (b) $t=5 T_{\text {ip }}\left(\mathcal{N}_{\mathcal{L}} \in[-0.46,0.54]\right)$, and (c) $t=6 T_{\mathrm{ip}}\left(\mathcal{N}_{\mathcal{L}} \in[-0.58\right.$, $0.28]$ ). Domain is $x, y \in[-\pi / 2, \pi / 2] c$. The PV contour $\varpi=-0.01$ (thick solid line) is included.

From the numerical point of view, some characteristics of the IGWs depend on the numerical resolution, since emission of shorter-wavelength IGWs is always possible as resolution is increased, notwithstanding how small the actual grid size is. Although we therefore ignore what might happen if even smaller scales are re- solved, the results show that the balanced flow prioritizes the emission of bursts of IGWs in the form of wave packets. A complete understanding of the mechanism responsible for this generation is still missing. We have suggested here that different mechanisms for spontaneous IGW generation may be at work.

One mechanism is related to the motion, in a loose way, of the vortex as an entity (which differs from the motion of the fluid particles in the vortex), and it is therefore related to the advection of $\mathrm{PV}$ or, equivalently, to the local rate of change of PV. The IGW packets related to the $\mathrm{PV}$ advection have a helical three-dimensional structure, similar to the PV filamentation, and the same sense of spiraling as the vortex.

Another mechanism is related to the fact that preexisting waves, obeying a common simple dynamics in the material description (i.e., in the cases analyzed here, the inertial waves experienced by fluid particles moving with the background vortex flow), can produce spatially coherent motion, eventually larger-scale horizontal divergence, and therefore larger-scale vertical motion, which in turns triggers the emission of new IGWs. Thus, in a certain sense, this second IGW emission is a vortex-IGW-vortex-IGW, or chain-effect, process, originally forced by the initially balanced vortex.

The spontaneous generation of IGWs may involve different mechanisms and be therefore a process more complex than initially expected. The large-scale vortical flow and the short-scale IGWs can coexist for long periods of time in the vortex interior, thus questioning the applicability of the strict separation between balanced and wave dynamics. Still, the aim is to unify these emission mechanisms into a single mechanism or mathematical theory. If the spontaneous emission of IGW is so frequent in the ocean it might play a relevant role in the transfer of energy from the balanced to the turbulent dissipative flow.

Acknowledgments. We acknowledge an FPU grant (AP2002-1895) and a research grant (CGL2005-01450/ CLI) from the Spanish Ministerio de Educación y Ciencia. We thank also two anonymous reviewers for their useful comments.

\section{REFERENCES}

Afanasyev, Y., 2003: Spontaneous emission of gravity waves by interacting vortex dipoles in a stratified fluid: Laboratory experiments. Geophys. Astrophys. Fluid Dyn., 97, 79-95.

Alford, M. H., and R. Pinkel, 2000: Observations of overturning in the thermocline: The context of ocean mixing. J. Phys. Oceanogr., 30, 805-832.

Baer, F., and J. J. Tribbia, 1977: On complete filtering of gravity modes through nonlinear initialization. Mon. Wea. Rev., 105, $1536-1539$. 
Daley, R., 1991: Atmospheric Data Analysis. Cambridge University Press, $457 \mathrm{pp}$.

Dritschel, D. G., and M. H. P. Ambaum, 1997: A contour-advective semi-Lagrangian algorithm for the simulation of finescale conservative fields. Quart. J. Roy. Meteor. Soc., 123, 1097-1130.

- and A. Viúdez, 2003: A balanced approach to modelling rotating stably stratified geophysical flows. J. Fluid Mech., 488, $123-150$.

Ford, R., 1994: Gravity wave radiation from vortex trains in rotating shallow water. J. Fluid Mech., 281, 81-118.

— M. E. McIntyre, and W. A. Norton, 2000: Balance and the slow quasimanifold: Some explicit results. J. Atmos. Sci., 57, 1236-1254.

Garrett, C., 1979: Mixing in the ocean interior. Dyn. Atmos. Oceans, 3, 239-265.

_ 2003: Internal tides and ocean mixing. Science, 301, 18581859.

Hoskins, B. J., 1975: The geostrophic momentum approximation and the semi-geostrophic equations. J. Atmos. Sci., 32, 233242.

Huang, X.-Y., and P. Lynch, 1993: Diabatic digital-filtering initialization: Application to the HIRLAM model. Mon. Wea. Rev., 121, 589-603.

Jacobs, S. J., 1991: Existence of a slow manifold in a model system of equations. J. Atmos. Sci., 48, 893-901.

Lane, T. P., J. D. Doyle, R. Plougonven, M. A. Shapiro, and R. D. Sharman, 2004: Observations and numerical simulations of inertia-gravity waves and shearing instabilities in the vicinity of a jet stream. J. Atmos. Sci., 61, 2692-2706.

Leith, C. E., 1980: Nonlinear normal mode initialization and quasi-geostrophic theory. J. Atmos. Sci., 37, 958-968.

Lighthill, M. J., 1952: On the sound generated aerodynamically. I. General theory. Proc. Roy. Soc. London, 211A, 564-587.

Lorenz, E. N., 1986: On the existence of a slow manifold. J. Atmos. Sci., 43, 1547-1557.

- - and V. Krishnamurthy, 1987: On the nonexistence of a slow manifold. J. Atmos. Sci., 44, 2940-2950.

Lovegrove, A. F., P. L. Read, and C. J. Richards, 2000: Generation of inertia-gravity waves in a baroclinically unstable fluid. Quart. J. Roy. Meteor. Soc., 126, 3233-3254.

Lynch, P., and X.-Y. Huang, 1992: Initialization of the HIRLAM model using a digital filter. Mon. Wea. Rev., 120, 1019-1034.

Machenhauer, B., 1977: On the dynamics of gravity wave oscillations in a shallow water model with application to normal mode initialization. Beitr. Phys. Atmos., 50, 253-271.
Plougonven, R., and V. Zeitlin, 2002: Internal gravity wave emission from a pancake vortex: An example of wave-vortex interaction in strongly stratified flows. Phys. Fluids, 14, 12591268 .

Rossby, C. G., 1938: On the mutual adjustment of pressure and velocity distributions in certain simple current systems. $J$. Mar. Res., 7, 239-263.

Saujani, S., and T. G. Shepherd, 2002: Comments on "Balance and the slow quasimanifold: Some explicit results." J. Atmos. Sci., 59, $2874-2877$.

Thorpe, S. A., 1999: On the breaking of internal waves in the ocean. J. Phys. Oceanogr., 29, 2433-2441.

Vanneste, J., and I. Yavneh, 2004: Exponentially small inertiagravity waves and the breakdown of quasigeostrophic balance. J. Atmos. Sci., 61, 211-223.

Vautard, R., and B. Legras, 1986: Invariant manifolds, quasigeostrophy and initialization. J. Atmos. Sci., 43, 565-584.

Viúdez, A., 2006: Spiral patterns of inertia-gravity waves in geophysical flows. J. Fluid Mech., 562, 73-82.

— , and D. G. Dritschel, 2003: Vertical velocity in mesoscale geophysical flows. J. Fluid Mech., 483, 199-223.

—_, and — , 2004a: Potential vorticity and the quasigeostrophic and semigeostrophic mesoscale vertical velocity. $J$. Phys. Oceanogr., 34, 865-887.

- , and - 2004b: Optimal potential vorticity balance of geophysical flows. J. Fluid Mech., 521, 343-352.

— and —, 2006: Spontaneous generation of inertia-gravity wave packets by balanced geophysical flows. J. Fluid Mech., 553, 107-117.

Warn, T., 1997: Nonlinear balance and quasi-geostrophic sets. Atmos.-Ocean, 35, 135-145.

Williams, P. D., P. L. Read, and T. W. N. Haine, 2003: Spontaneous generation and impact of inertia-gravity waves in a stratified, two-layer shear flow. Geophys. Res. Lett., 30, 2255, doi:10.1029/2003GL018498.

, T. W. N. Haine, and P. L. Read, 2005: On the generation mechanism of short-scale unbalanced modes in rotating twolayer flows with vertical shear. J. Fluid Mech., 528, 1-22.

Zhang, F., 2004: Generation of mesoscale gravity waves in uppertropospheric jet-front systems. J. Atmos. Sci., 61, 440-457.

- S. E. Koch, C. A. Davis, and M. L. Kaplan, 2001: Wavelet analysis and the governing dynamics of a large amplitude gravity wave event along the east coast of the United States. Quart. J. Roy. Meteor. Soc., 127, 2209-2245. 